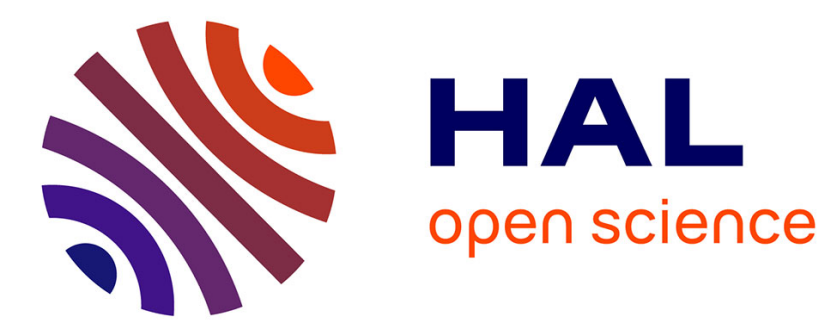

\title{
Model-free fractional order differentiator based on fractional order Jacobi orthonormal functions
} Xiao-Lin Li, Yi-Ming Chen, Da-Yan Liu, Yan-Qiao Wei, Driss Boutat

\section{To cite this version:}

Xiao-Lin Li, Yi-Ming Chen, Da-Yan Liu, Yan-Qiao Wei, Driss Boutat. Model-free fractional order differentiator based on fractional order Jacobi orthonormal functions. Digital Signal Processing, 2017, 71, pp.69-82. 10.1016/j.dsp.2017.09.001 . hal-02884925

\section{HAL Id: hal-02884925 https://hal.science/hal-02884925}

Submitted on 30 Jun 2020

HAL is a multi-disciplinary open access archive for the deposit and dissemination of scientific research documents, whether they are published or not. The documents may come from teaching and research institutions in France or abroad, or from public or private research centers.
L'archive ouverte pluridisciplinaire HAL, est destinée au dépôt et à la diffusion de documents scientifiques de niveau recherche, publiés ou non, émanant des établissements d'enseignement et de recherche français ou étrangers, des laboratoires publics ou privés. 


\title{
Model-free fractional order differentiator based on fractional order Jacobi orthonormal functions
}

\author{
Xiao-Lin Li ${ }^{\text {a }}$, Yi-Ming Chen ${ }^{\text {a,b }}$, Da-Yan Liu ${ }^{c}$, Yan-Qiao Wei $^{c}$, Driss Boutat $^{\mathrm{c}}$ \\ ${ }^{a}$ College of Sciences, Yanshan University, Qinhuangdao, 066004, Hebei, China \\ ${ }^{\mathrm{b}}$ LE STUDIUM RESEARCH PROFESSOR, Loire Valley Institute for Advanced Studies, Bourges, France PRISME \\ (INSA-Institut National des sciences appliquées/University of Orleans) -88, Boulvevard Lahitolle, 18000 Bourges, France
}

${ }^{\mathrm{c}}$ INSA Centre Val de Loire, Université d'Orléans, PRISME EA 4229, Bourges Cedex 18022, France

\begin{abstract}
The aim of this paper is to design an algebraic and robust fractional order differentiator to estimate both the Riemann-Liouville and the Caputo fractional derivatives with an arbitrary order of an unknown signal in noisy environment, without knowing the model defining the signal. For this purpose, a new class of fractional order Jacobi orthonormal functions is firstly introduced. Secondly, the truncated fractional order Jacobi orthonormal series expansion is applied to filter the noisy signal, whose fractional derivative is used to estimate the desired one. Thus, the obtained differentiator is exactly given by an integral formula which depends on a set of design parameters. Thirdly, by applying the generalized Taylor's formula, some error analysis is provided. In particular, error bounds are given, which permit to study the design parameters' influence. Fourthly, a digital fractional order differentiator is deduced in discrete noisy case. Finally, by comparing with two existing fractional order differentiators, numerical results are given to illustrate the accuracy and the robustness of the proposed fractional order differentiator.
\end{abstract}

Key words: Fractional order differentiator, Fractional order Jacobi orthonormal functions, Error analysis.

\section{Introduction}

Fractional calculus was introduced in many fields of science and engineering long time ago. It was first develope$\mathrm{d}$ by mathematicians in the middle of the ninetieth century [1]. During the past decades, fractional calculus has gained great interest in many scientific and engineering fields, including control, flow propagation, signal processing, image processing, electrical networks, and etc. [2-9]. In most cases, the fractional derivatives of a signal can not be analytically calculated. Moreover, when the signal is a unknown, it is usually measured in noisy environment. In order to estimate the fractional derivatives of an unknown signal from its discrete noisy observation, various robust fractional order differentiators have been designed in the frequency domain [10-12] and in the time domain [13-18]. Among them, there exists

\footnotetext{
* Corresponding author D.Y. Liu.

Email addresses: 150335831750163.com (Xiao-Lin Li), chenym@ysu.edu.cn (Yi-Ming Chen), dayan.liu@insa-cvl.fr (Da-Yan Liu), yanqiao.wei@insa-cvl.fr (Yan-Qiao Wei), driss.boutat@insa-cvl.fr (Driss Boutat).
}

a class of model-free differentiators, which are obtained from the truncation of an analytical expression, without considering the model of the studied signal $[10,11,13,14]$.

Recently, the model-free fractional order Jacobi differentiator was obtained by the fractional order differentiation by integration method [14], as the extension of the integer order one [19-23]. This method is algebraic, where the differentiator was exactly given by an integral formula. Moreover, it is robust against corrupting noises thanks to the integral [24]. The idea of this method is to filter the studied noisy signal by the Jacobi polynomial filter whose fractional derivative is used to approximate the one of the signal. Since the fractional derivative of a polynomial is a power function, the desired fractional derivative is approximated by a power function. Consequently, an intuitive idea is to approximate the studied signal by a power function whose fractional derivative is again a power function. Similar idea was used to solve fractional order differential equations in $[25,26]$. Bearing these ideas in mind, the aim of this paper is to extend the fractional order differentiation by integration method by introducing fractional order Jacobi orthonormal functions to filter the studied noisy signal, which 
are power functions and whose fractional derivatives are also power functions.

The contributions of the paper are outlined as follows:

(1) the fractional order differentiation by integration method [14] is extended using fractional order Jacobi orthonormal functions to estimate both the Riemann-Liouville and the Caputo fractional derivatives for noisy signals,

(2) some analysis and error bounds are given, which permit to study the design parameters' influence,

(3) numerical algorithms are provided in discrete noisy case, which are robust against the numerical errors produced by negative design parameters' values,

(4) the proposed differentiator does not produce any truncated term error for power functions. Moreover, it will be shown in numerical simulations that the proposed differentiator can improve the one in [14].

This paper is organized as follows. In Section 2, some useful definitions and properties on fractional calculus and fractional order Jacobi orthonormal functions are given. The main results are given in Section 3. An algebraic fractional order differentiator is proposed. Then, some error analysis is provided. Moreover, four numerical algorithms are given in discrete noisy case. In Section 4, numerical results illustrate the accuracy and the robustness of the proposed fractional order differentiator. Finally, conclusions are summarized in Section 5.

\section{Preliminaries}

In this section, some useful definitions and properties on fractional calculus are given. Moreover, the fractional order Jacobi orthonormal functions are introduced with some useful properties.

\subsection{Fractional calculus}

Let $I=[a, b] \subset \mathbb{R}^{1}, \alpha \in \mathbb{R}_{+}$, and $l=\lceil\alpha\rceil$, where $\lceil\alpha\rceil$ denotes the smallest integer larger than or equal to $\alpha$. Then, the following definitions can be found in [3] and [4].

Definition 1 The $\alpha$ order Riemann-Liouville fractional integral of a function $y$ is defined on $] a, b]$ as follows:

$$
\left\{\begin{aligned}
\mathrm{D}_{a, t}^{0} y(t) & :=y(t) \\
\mathrm{D}_{a, t}^{-\alpha} y(t) & :=\frac{1}{\Gamma(\alpha)} \int_{a}^{t}(t-\tau)^{\alpha-1} y(\tau) d \tau
\end{aligned}\right.
$$

\footnotetext{
1 In this paper, $\mathbb{N}^{*}$ (resp. $\mathbb{R}_{+}^{*}$ ) denotes the set of strictly positive integers (resp. strictly positive real numbers), $\mathbb{R}_{+}$ denotes the set of positive real numbers, and $\mathbb{Z}_{-}$denotes the set of negative integers (including 0 ).
}

where $\Gamma(\cdot)$ is the well-known Gamma function [27].

Definition 2 The $\alpha$ order Riemann-Liouville fractional derivative of a function $y$ is defined on $] a, b]$ as follows:

$$
{ }_{R} \mathrm{D}_{a, t}^{\alpha} y(t):=\frac{d^{l}}{d t^{l}}\left\{\mathrm{D}_{a, t}^{\alpha-l} y(t)\right\}
$$

Definition 3 The $\alpha$ order Caputo fractional derivative of a function $y$ is defined on ] a,b] as follows:

$$
{ }_{C} \mathrm{D}_{a, t}^{\alpha} y(t):=\mathrm{D}_{a, t}^{\alpha-l}\left\{y^{(l)}(t)\right\} .
$$

By applying integration by parts to (2), the following result can be obtained.

Lemma 1 [3] The relation between the RiemannLiouville and the Caputo fractional derivatives is give as follows: $\forall \alpha \in \mathbb{R}_{+} \backslash \mathbb{N}$,

$$
{ }_{R} \mathrm{D}_{a, t}^{\alpha} y(t)=\sum_{i=0}^{\lceil\alpha\rceil-1} \frac{(t-a)^{i-\alpha}}{\Gamma(i+1-\alpha)} y^{(i)}(a)+{ }_{C} \mathrm{D}_{a, t}^{\alpha} y(t) .
$$

Using (2) and (3), the fractional derivatives of a power function can be obtained in the following lemma.

Lemma 2 Let $y(\cdot)=(\cdot-a)^{n \alpha}$ with $\left.\left.\alpha \in\right] 0,1\right], n \in \mathbb{N}$ and $m \in \mathbb{N}^{*}$, then we have: $\forall t>a$,

$$
\begin{aligned}
{ }_{R} \mathrm{D}_{a, t}^{m \alpha} y(t) & =\frac{\Gamma(n \alpha+1)}{\Gamma((n-m) \alpha+1)}(t-a)^{(n-m) \alpha}, \\
{ }_{C} \mathrm{D}_{a, t}^{m \alpha} y(t) & =\left\{\begin{array}{l}
0, \quad \text { if } n \alpha \in \mathbb{N} \text { and } n \alpha<\lceil m \alpha\rceil, \\
\frac{\Gamma(n \alpha+1)}{\Gamma((n-m) \alpha+1)}(t-a)^{(n-m) \alpha}, \text { else. }
\end{array}\right.
\end{aligned}
$$

In order to simplify the notations in the sequel, $\mathrm{D}_{a, t}^{\alpha} y$ ( $\alpha \in \mathbb{R}_{+}$) is used to denote both the Riemann-Liouville and the Caputo fractional derivatives. Then, the following lemma is given.

Lemma 3 [2,3] The fractional derivatives satisfy the following properties with $\alpha \in \mathbb{R}$,

- Scale change: Let $\lambda \in \mathbb{R}_{+}^{*}$, then we have: $\forall t>a$,

$$
\mathrm{D}_{a, \lambda t}^{\alpha} y(\lambda t)=\frac{1}{\lambda^{\alpha}} \mathrm{D}_{\frac{a}{\lambda}, t}^{\alpha} y(\lambda t) .
$$

- Transition: Let $a \in \mathbb{R}$, then we have: $\forall t>0$,

$$
\mathrm{D}_{0, t}^{\alpha} y(a+t)=\mathrm{D}_{a, a+t}^{\alpha} y(t) .
$$


- Additive index law: Let $\beta \in \mathbb{R}_{+}$. If $\lceil\beta\rceil<\alpha$, we have: $\forall t>a$,

$$
\mathrm{D}_{a, t}^{\beta}\left\{\mathrm{D}_{a, t}^{-\alpha} y(t)\right\}=\mathrm{D}_{a, t}^{\beta-\alpha} y(t) .
$$

Finally, this subsection ends with the following definition and theorem.

Definition 4 [4] Let $k \in \mathbb{N}$, the Caputo fractional sequential derivative of a function $y$ is defined as follows:

$$
{ }_{C} \mathcal{D}_{a, t}^{k \alpha} y(t):= \begin{cases}y(t), & \text { for } k=0, \\ { }_{C} \mathrm{D}_{a, t}^{\alpha}\left\{{ }_{C} \mathcal{D}_{a, t}^{(k-1) \alpha} y(t)\right\}, & \text { for } k \geq 1 .\end{cases}
$$

\section{Theorem 1 [28] Generalized Taylor's Formula}

Let $\alpha \in] 0,1]$ and $N \in \mathbb{N}$. Assume that ${ }_{C} \mathcal{D}_{a, t}^{i \alpha} y$ is continuous on $] a, b]$, for $i=0,1, \cdots, N+1$. Then, we have: $\forall t \in] a, b]$,

$$
\begin{aligned}
y(t)= & \sum_{i=0}^{N} \frac{(t-a)^{i \alpha}}{\Gamma(i \alpha+1)}{ }_{C} \mathcal{D}_{a, t}^{k \alpha} y(a) \\
& +\mathrm{D}_{a, t}^{-(N+1) \alpha}\left\{{ }_{C} \mathcal{D}_{a, t}^{(N+1) \alpha} y(t)\right\} .
\end{aligned}
$$

\subsection{Fractional order Jacobi orthonormal functions}

Based on the shifted Jacobi orthogonal polynomials [27], a class of power functions can be introduced in the following definition.

Definition 5 Let $\alpha \in] 0,1]$ and $n \in \mathbb{N}$, then the fractional order Jacobi function is defined on $[0,1]$ as follows:

$$
L_{n, \alpha}^{(\mu, \kappa)}(\tau):=\sum_{j=0}^{n} C_{n+\mu}^{j} C_{n+\kappa}^{n-j}\left(\tau^{\alpha}-1\right)^{n-j} \tau^{j \alpha},
$$

where $\mu, \kappa \in]-1,+\infty[$, and the generalized binomial coefficients are used: $C_{a}^{b}=\frac{\Gamma(a+1)}{\Gamma(b+1) \Gamma(a-b+1)}$ with $a, b \in$ $\mathbb{R} \backslash \mathbb{Z}_{-}$.

In fact, $L_{n, \alpha}^{(\mu, \kappa)}$ is defined by taking $L_{n, \alpha}^{(\mu, \kappa)}(\tau)=$ $P_{n}^{(\mu, \kappa)}\left(\tau^{\alpha}\right)$, where $P_{n}^{(\mu, \kappa)}$ is the $n^{\text {th }}$ order shifted Jacobi polynomial defined on $[0,1]$. In particular, if $\alpha=1$, we have $L_{n, \alpha}^{(\mu, \kappa)} \equiv P_{n}^{(\mu, \kappa)}$.

Let $g_{1}, g_{2} \in \mathcal{C}([0,1])$, where $\mathcal{C}([0,1])$ refers to the space of functions continuous on the interval $[0,1]$, the scalar product $\left\langle g_{1}, g_{2}\right\rangle$ is defined by:

$$
\left\langle g_{1}, g_{2}\right\rangle:=\int_{0}^{1} g_{1}(\tau) g_{2}(\tau) \phi_{\alpha}^{(\mu, \kappa)}(\tau) d \tau
$$

with respect to the following weight function:

$$
\phi_{\alpha}^{(\mu, \kappa)}(\tau):=\alpha\left(1-\tau^{\alpha}\right)^{\mu} \tau^{\alpha(\kappa+1)-1} .
$$

Then, by applying the change of variables: $\tau \rightarrow \tau^{\alpha}$ and using the norm of $P_{n}^{(\mu, \kappa)}$ [14], we get:

$\left\|L_{n, \alpha}^{(\mu, \kappa)}\right\|^{2}=\frac{\Gamma(\mu+n+1) \Gamma(\kappa+n+1)}{\Gamma(\mu+\kappa+n+1) \Gamma(n+1)(2 n+\mu+\kappa+1)}$.

In order to simplify the notations in the sequel, for fixed $\alpha, \mu$ and $\kappa$, we define: $\forall n \in \mathbb{N}$,

$$
E_{n}:=\frac{L_{n, \alpha}^{(\mu, \kappa)}}{\left\|L_{n, \alpha}^{(\mu, \kappa)}\right\|} .
$$

Then, the following properties of the fractional order Jacobi functions $\left\{E_{n}\right\}_{n \in \mathbb{N}}$ can be deduced from the ones of the shifted Jacobi polynomials.

Firstly, thanks to the orthogonality of the shifted Jacobi polynomials, the following lemma can be obtained.

Lemma 4 Under the scalar product $\langle\cdot, \cdot\rangle$ defined in (13) with respect to the weight function $\phi_{\alpha}^{(\mu, \kappa)}(\tau)$ defined in (14), we have:

- the fractional order Jacobi functions $\left\{E_{n}\right\}_{n \in \mathbb{N}}$ are orthonormal,

- $\forall n \in \mathbb{N}^{*}, E_{n}$ is orthogonal to $l_{m, \tau}(\tau)=\tau^{m \alpha}$ for $m=$ $0, \cdots, n-1$.

Proof. This proof can be completed by applying the change of variables: $\tau \rightarrow \tau^{\alpha}$ and using the orthogonal properties of $P_{n}^{(\mu, \kappa)}$ [14].

Secondly, thanks to the completeness of the shifted Jacobi orthogonal polynomials [29], the following lemma is provided.

Lemma 5 Let $y \in \mathcal{C}(I)$ with $I=[a, b]$ and $h=b-$ $a$, then the fractional order Jacobi orthonormal series expansion of $y(a+h \cdot)$ is given by: $\forall \xi \in[0,1]$,

$$
y(a+h \xi)=\sum_{i=0}^{+\infty}\left\langle E_{i}(\cdot), y(a+h \cdot)\right\rangle E_{i}(\xi) .
$$

In particular, if $y$ is a power function defined on I with the following form:

$$
y(\tau)=\sum_{i=0}^{n} a_{i}(\tau-a)^{i \alpha},
$$


where $\alpha \in] 0,1], n \in \mathbb{N}$ and $a_{i} \in \mathbb{R}$ for $i=0, \ldots, n$, then we have: $\forall \xi \in[0,1]$,

$$
y(a+h \xi)=\sum_{i=0}^{n}\left\langle E_{i}(\cdot), y(a+h \cdot)\right\rangle E_{i}(\xi) .
$$

Proof. (17) can be obtained by applying the change of variables: $\tau \rightarrow \tau^{\alpha}$ in the Jacobi orthogonal series expansion of $y(a+h \cdot)$ [14]. Then, this proof can be completed by applying Lemma 4 and (18) in (17).

Finally, by applying Lemma 2 to (12) and applying the well-known binomial theorem, the fractional derivatives of the fractional order Jacobi orthonormal functions can be obtained in the following lemma.

Lemma 6 Let $\alpha \in] 0,1]$, then the mo order derivatives of the fractional order Jacobi orthonormal function is given as follows: $\forall \tau \in] 0,1]$,

$$
\begin{aligned}
{ }_{R} \mathrm{D}_{0, \tau}^{m \alpha} E_{n}(\tau)= & \frac{1}{\left\|L_{n, \alpha}^{(\mu, \kappa)}\right\|}\left\{\sum_{j=0}^{n} \sum_{i=0}^{n-j} \frac{\Gamma((n-i) \alpha+1)}{\Gamma((n-i-m) \alpha+1)}\right. \\
& \left.\times c_{\mu, \kappa, n, j, i} \tau^{(n-i-m) \alpha}\right\},
\end{aligned}
$$

$$
\begin{aligned}
{ }_{C} \mathrm{D}_{0, \tau}^{m \alpha}\left\{E_{n}(\tau)\right\}= & \frac{1}{\left\|L_{n, \alpha}^{(\mu, \kappa)}\right\|}\left\{\sum_{j=0}^{n} \sum_{i=0}^{n-j} \frac{\Gamma((n-i) \alpha+1)}{\Gamma((n-i-m) \alpha+1)}\right. \\
& \left.\times d_{\alpha, n, i, m} c_{\mu, \kappa, n, j, i} \tau^{(n-i-m) \alpha}\right\},
\end{aligned}
$$

where $c_{\mu, \kappa, n, j, i}=(-1)^{i} C_{n+\mu}^{j} C_{n+\kappa}^{n-j} C_{n-j}^{i},\left\|L_{n, \alpha}^{(\mu, \kappa)}\right\| i s$ given by (15), and $d_{\alpha, n, i, m}=0$ if $(n-i) \alpha \in \mathbb{N}$ and $(n-i) \alpha<\lceil m \alpha\rceil, d_{\alpha, n, i, m}=1$ else.

\section{Main results}

Let $y \in \mathcal{C}(\mathbb{R})$, and $y^{\varpi}$ be a noisy observation of $y$ on an interval $I=[a, b] \subset \mathbb{R}$ of length $h=b-a: \forall t \in I$,

$$
y^{\varpi}(t)=y(t)+\varpi(t),
$$

where the noise $\varpi$ is integrable and locally essentially bounded, i.e. locally bounded except on a set of measure zero.

The objective is to estimate the fractional derivatives of $y$ using the observation signal $y^{\varpi}$. For this purpose, the fractional order differentiation by integration method proposed in [14] will be extended by using fractional order Jacobi orthonormal functions.

\subsection{Generalized fractional order Jacobi differentiator}

For a moment, let us ignore the noise. Then, we define the following approximation of $y$ by taking its $N^{t h}$ order truncated fractional order Jacobi orthonormal series expansion: $\forall \xi \in[0,1]$,

$y(a+h \xi) \approx D_{N}^{0} y(a+h \xi):=\sum_{i=0}^{N}\left\langle E_{i}(\cdot), y(a+h \cdot)\right\rangle E_{i}(\xi)$.

Thus, $y$ is approximated by the power function $D_{N}^{0} y(a+$ $h \cdot)$. In the case of $\alpha=1, D_{N}^{0} y(a+h \cdot)$ is the Jacobi polynomial filter, whose fractional derivative is a power function used to construct the fractional order Jacobi differentiator [14]. Inspired by this idea, the following definition is introduced.

Definition 6 Let $m \in \mathbb{N}$ and $\alpha \in] 0,1]$, the generalized fractional order Jacobi differentiator $D_{N}^{m \alpha} y(a+h \cdot)$ is defined as the $m \alpha$ order derivative of $D_{N}^{0} y(a+\cdot)$ defined in (23), i.e. we have: $\forall \xi \in] 0,1]$,

$\mathrm{D}_{0, h \xi}^{m \alpha} y(a+h \xi) \approx D_{N}^{m \alpha} y(a+h \xi):=\mathrm{D}_{0, h \xi}^{m \alpha}\left\{D_{N}^{0} y(a+h \xi)\right\}$.

This differentiator specifies ${ }_{R} D_{N}^{m \alpha} y^{\varpi}(a+h \xi)$ (resp. $\left.{ }_{C} D_{N}^{m \alpha} y^{\varpi}(a+h \xi)\right)$ when it is used to estimate ${ }_{R} \mathrm{D}_{0, h \xi}^{m \alpha} y(a+h \xi)\left(\operatorname{resp} .{ }_{C} \mathrm{D}_{0, h \xi}^{m \alpha} y(a+h \xi)\right)$.

Consequently, according to Lemma $6, D_{N}^{m \alpha} y(a+h \cdot)$ is also a power function. Moreover, according to Lemma 5 and Definition 6, the fractional derivative of a power function can be exactly obtained by $D_{N}^{m \alpha} y(a+h \cdot)$. This result is given in the following lemma.

Lemma 7 If $y$ is a power function defined by the form given in (18) with $n \leq N$, then we have: $\forall \xi \in] 0,1]$,

$$
D_{N}^{m \alpha} y(a+h \xi)=\mathrm{D}_{0, h \xi}^{m \alpha} y(a+h \xi) .
$$

Let us consider the noise in the sequel. Then, the generalized fractional order Jacobi differentiator is given in the noisy environment by the following proposition.

Proposition 1 The generalized fractional order Jacobi differentiator $D_{N}^{m \alpha} y(a+h \cdot)$ can be given by an integral formula as follows: $\forall \xi \in] 0,1]$,

$$
D_{N}^{m \alpha} y(a+h \xi)=\frac{1}{h^{m \alpha}} \int_{0}^{1} Q_{N}(\tau, \xi) y(a+h \tau) d \tau,
$$

where $\left.h \in \mathbb{R}_{+}^{*}, N \in \mathbb{N}, \mu, \kappa \in\right]-1,+\infty[$, and

$$
Q_{N}(\tau, \xi)=\phi_{\alpha}^{(\mu, \kappa)}(\tau) \sum_{i=0}^{N} E_{i}(\tau) \mathrm{D}_{0, \xi}^{m \alpha}\left\{E_{i}(\xi)\right\}
$$


with $\phi_{\alpha}^{(\mu, \kappa)}(\tau)=\alpha\left(1-\tau^{\alpha}\right)^{\mu} \tau^{\alpha(\kappa+1)-1}$. Moreover, let $y^{\varpi}$ be the noisy observation defined in (22), then $\mathrm{D}_{0, h \xi}^{m \alpha} y(a+$ $h \xi)$ can be estimated by applying the generalized fractional order Jacobi differentiator $D_{N}^{m \alpha} y^{\varpi}(a+h \xi)$ defined in the noisy case as follows: $\forall \xi \in] 0,1]$,

$$
D_{N}^{m \alpha} y^{\varpi}(a+h \xi):=\frac{1}{h^{m \alpha}} \int_{0}^{1} Q_{N}(\tau, \xi) y^{\varpi}(a+h \tau) d \tau .
$$

Proof. By applying the scale change given in (7) to (24), we obtain: $\forall \xi \in] 0,1]$,

$$
D_{N}^{m \alpha} y(a+h \xi)=\frac{1}{h^{m \alpha}} \mathrm{D}_{0, \xi}^{m \alpha}\left\{D_{N}^{0} y(a+h \xi)\right\} .
$$

Then, by applying the linearity of the fractional derivative operator, we get:

$$
\begin{aligned}
D_{N}^{m \alpha} y(a+h \xi) & =\frac{1}{h^{m \alpha}} \sum_{i=0}^{N}\left\langle E_{i}(\cdot), y(a+h \cdot)\right\rangle \mathrm{D}_{0, \xi}^{m \alpha}\left\{E_{i}(\xi)\right\} \\
& =\frac{1}{h^{m \alpha}} \int_{0}^{1} Q_{N}(\tau, \xi) y(a+h \tau) d \tau
\end{aligned}
$$

Finally, this proof can be completed by substituting $y$ by $y^{\varpi}$ in $(30)$.

Remark 1 By applying the translation property given in (8), we get: $\mathrm{D}_{0, h \xi}^{m \alpha} y(a+h \xi)=\mathrm{D}_{a, a+h \xi}^{m \alpha} y(h \xi)$. Consequently, $D_{N}^{m \alpha} y^{\varpi}(a+\tau)$ is actually used to estimate $\mathrm{D}_{a, a+\tau}^{m \alpha} y(\tau)$ with $\tau=h \xi$.

Consequently, according to (28), the proposed differentiator $D_{N}^{m \alpha} y^{\varpi}(a+h \xi)$ is exactly given by an integral formula. Hence, it is an algebraic differentiator. Moreover, the integral can be considered as a low-pass filter in noisy cases [24]. Since the integral involves fractional order Jacobi functions, this method is called fractional order differentiation by integration based on fractional order Jacobi orthonormal functions.

$D_{N}^{m \alpha} y^{\varpi}(a+h \xi)$ can be used to estimate both the Riemann-Liouville and the Caputo fractional derivatives of $y$ with an arbitrary order. Indeed, $\forall \alpha^{*} \in \mathbb{R}$, there exists $\alpha \in] 0,1]$ and $m \in \mathbb{N}$ such that $\alpha^{*}=m \alpha$. Moreover, $D_{N}^{m \alpha} y^{\varpi}(a+h \xi)$ is a model-free differentiator which can be used for different situations without knowing the model defining $y$. Similar to the fractional order Jacobi differentiator, the proposed differentiator can be used for both continuous-time and discrete-time signals in both off-line and on-line applications [14].

Finally, it can be seen that the generalized fractional order Jacobi differentiator $D_{N}^{m \alpha} y^{\varpi}(a+h \xi)$ depends on a set of parameters $\{m, \alpha, h, \mu, \kappa, N\}$ :
- $m \in \mathbb{N}$ and $\alpha \in] 0,1]$ are determined by $m \alpha$ which is the order of the required fractional derivative,

- $h \in \mathbb{R}_{+}$is the length of the interval where the noisy signal is observed,

- $\mu, \kappa \in]-1,+\infty[$ are the design parameters in the fractional order Jacobi functions,

- $N \in \mathbb{N}$ is the truncation order in the truncated fractional order Jacobi orthonormal series expansions.

In order to give a guideline on how to choose these parameters, the estimation error for $D_{N}^{m \alpha} y^{\varpi}(a+h \xi)$ is studied in the next subsection.

\subsection{Error analysis in continuous noisy case}

The estimation error for the fractional order Jacobi differentiator has been studied in [14], where some error bounds were obtained. Using a more general way, the error analysis for the proposed generalized fractional order Jacobi differentiator is provided in this subsection.

The estimation error for the generalized fractional order Jacobi differentiator in continuous noisy case, denoted by $e_{C}(\xi):=D_{N}^{m \alpha} y^{\varpi}(a+h \xi)-\mathrm{D}_{0, h \xi}^{m \alpha} y(a+h \xi)$, is the sum of the following two errors $e^{\varpi}(\xi)$ and $e_{N}^{\infty}(\xi)$ which are:

- the noise error contribution $e^{\varpi}(\xi):=D_{N}^{m \alpha} y^{\varpi}(a+$ $h \xi)-D_{N}^{m \alpha} y(a+h \xi)$, which is due to the noise in the signal $y^{\varpi}$,

- the $N^{t h}$ order truncated term error $e_{N}^{\infty}(\xi):=$ $D_{N}^{m \alpha} y(a+h \xi)-\mathrm{D}_{0, h \xi}^{m \alpha} y(a+h \xi)$, which comes from the truncated fractional order Jacobi orthonormal series expansion of $y$ in $(23)$.

The previous two errors are studied as follows. Firstly, we begin with the noise error contribution.

Proposition 2 Let $y^{\varpi}$ be the noisy observation defined in (22) and $D_{N}^{m \alpha} y^{\varpi}(a+h \xi)$ be the generalized fractional order Jacobi differentiator proposed in Proposition 1. Then, the noise error contribution in $D_{N}^{m \alpha} y^{\varpi}(a+h \xi)$ is given as follows: $\forall \xi \in] 0,1]$,

$$
e^{\varpi}(\xi)=\frac{1}{h^{m \alpha}} \int_{0}^{1} Q_{N}(\tau, \xi) \varpi(a+h \tau) d \tau,
$$

where $\alpha \in] 0,1], h \in \mathbb{R}_{+}^{*}, Q_{N}(\cdot, \cdot)$ is given by (27). Moreover, if the essential supremum ${ }^{2}$ of $|\varpi|$ exists, i.e. $\delta=$ ess sup $|\varpi|<\infty$, then we have: $\forall \xi \in] 0,1]$,

$$
\left|e^{\varpi}(\xi)\right| \leq \frac{\delta}{h^{m \alpha}} E_{\mu, \kappa, m, \alpha, N}(\xi)
$$

where $E_{\mu, \kappa, m, \alpha, N}(\xi)=\int_{0}^{1}\left|Q_{N}(\tau, \xi)\right| d \tau$.

\footnotetext{
2 The essential supremum of $|\varpi|$, denoted by ess sup $|\varpi|$, is defined as follows: ess sup $|\varpi|:=\inf \{c \in \mathbb{R}: \eta(\{t \in \mathbb{R}$ : $|\varpi(t)|>c\})=0\}$, where $\eta$ is a measure on $\mathbb{R}$.
} 
Proof. Using $y^{\varpi}=y+\varpi$ in (28), the noise error contribution for $D_{N}^{m \alpha} y^{\varpi}(a+h \cdot)$ can be obtained as follows: $\forall \xi \in] 0,1]$,

$$
\begin{aligned}
e^{\varpi}(\xi) & =D_{N}^{m \alpha} y^{\varpi}(a+h \xi)-D_{N}^{m \alpha} y(a+h \xi) \\
& =\frac{1}{h^{m \alpha}} \int_{0}^{1} Q_{N}(\tau, \xi) \varpi(a+h \tau) d \tau .
\end{aligned}
$$

Then, this proof can be completed by taking the absolute value of $e^{\varpi}(\xi)$.

Secondly, the truncated term error is studied.

Theorem 2 Let $D_{N}^{m \alpha} y^{\varpi}(a+h \xi)$ be the generalized fractional order Jacobi differentiator proposed in Proposition 1. If $m<N$ and ${ }_{C} \mathcal{D}_{a, t}^{i \alpha} f$ is continuous on $\left.] a, b\right]$, for $i=0,1, \cdots, N+1$, the $N^{\text {th }}$ order truncated term error for $D_{N}^{m \alpha} y^{\varpi}(a+h \xi)$ can be given as follows: $\left.\left.\forall \xi \in\right] 0,1\right]$,

$$
e_{N}^{\infty}(\xi)=h^{(N+1-m) \alpha}\left(\int_{0}^{1} Q_{N}(\tau, \xi) I_{1}(\tau) d \tau+I_{2}(\xi)\right)
$$

where $\alpha \in] 0,1], h \in \mathbb{R}_{+}^{*}, Q_{N}(\cdot, \cdot)$ is given by (27), and

$$
\begin{aligned}
I_{1}(\tau)= & \frac{\tau^{(N+1) \alpha}}{\Gamma((N+1) \alpha)} \int_{0}^{1}(1-s)^{(N+1) \alpha-1} \\
& \times{ }_{C} \mathcal{D}_{0, h \tau s}^{(N+1) \alpha} y(a+h \tau s) d s, \\
I_{2}(\xi)= & \frac{\xi^{(N+1-m) \alpha}}{\Gamma((N+1-m) \alpha)} \int_{0}^{1}(1-s)^{(N+1-m) \alpha-1} \\
& \times{ }_{C} \mathcal{D}_{0, h \xi s}^{(N+1) \alpha} y(a+h \xi s) d s .
\end{aligned}
$$

Moreover, if $M_{N+1}=\sup \left\{\left|{ }_{C} \mathcal{D}_{a, t}^{(N+1) \alpha} y(t)\right|, t \in I\right\}$ exists, then $e_{N}^{\infty}(\cdot)$ is bounded as follows: $\left.\left.\forall \xi \in\right] 0,1\right]$,

$$
\left|e_{N}^{\infty}(\xi)\right| \leq h^{(N+1-m) \alpha} M_{N+1} C_{\mu, \kappa, m, \alpha, N}(\xi),
$$

where $C_{\mu, \kappa, m, \alpha, N}(\xi)=\frac{I_{3}(\xi)}{\Gamma((N+1) \alpha+1)}+\frac{\xi^{(N+1-m) \alpha}}{\Gamma((N+1-m) \alpha+1)}$ with $I_{3}(\xi)=\int_{0}^{1}\left|Q_{N}(\tau, \xi) \tau^{(N+1) \alpha}\right| d \tau$.

Proof. By applying the Generalized Taylor's Formula given in Theorem 1 to $y$, we get: $\forall \xi \in] 0,1]$,

$y(a+h \xi)=y_{N}(a+h \xi)+\mathrm{D}_{0, h \xi}^{-(N+1) \alpha}\left\{C_{C} \mathcal{D}_{0, h \xi}^{(N+1) \alpha} y(a+h \xi)\right\}$

where

$$
y_{N}(a+h \xi):=\sum_{i=0}^{N} \frac{(h \xi)^{i \alpha}}{\Gamma(i \alpha+1)} C^{\mathcal{D}_{0, h \xi}^{k \alpha} y(a) .}
$$

Then, let us divide the truncated term error into the following two parts: $\forall \xi \in] 0,1]$,

$$
\begin{aligned}
e_{N}^{\infty}(\xi):= & D_{N}^{m \alpha} y(a+h \xi)-\mathrm{D}_{0, h \xi}^{m \alpha} y(a+h \xi) \\
= & \left(D_{N}^{m \alpha} y(a+h \xi)-\mathrm{D}_{0, h \xi}^{m \alpha} y_{N}(a+h \xi)\right) \\
& +\left(\mathrm{D}_{0, h \xi}^{m \alpha} y_{N}(a+h \xi)-\mathrm{D}_{0, h \xi}^{m \alpha} y(a+h \xi)\right)
\end{aligned}
$$

On the one hand, by applying Lemma 7 and Proposition 1, we get:

$$
\begin{aligned}
& \left.D_{N}^{m \alpha} y(a+h \xi)-\mathrm{D}_{0, h \xi}^{m \alpha} y_{N}(a+h \xi)\right) \\
& =\frac{1}{h^{m \alpha}} \int_{0}^{1} Q_{N}(\tau, \xi)\left(y(a+h \tau)-y_{N}(a+h \tau)\right) d \tau .
\end{aligned}
$$

Hence, using (37) and (38) in (40) and by applying the scale change property given in (7), we obtain:

$$
\begin{aligned}
& \left.D_{N}^{m \alpha} y(a+h \xi)-D_{0, h \xi}^{m \alpha} y_{N}(a+h \xi)\right) \\
& =h^{(N+1-m) \alpha} \int_{0}^{1} Q_{N}(\tau, \xi) I_{1}(\tau) d \tau
\end{aligned}
$$

where $I_{1}(\tau)=\mathrm{D}_{0, \tau}^{-(N+1) \alpha}\left\{{ }_{C} \mathcal{D}_{0, h \tau}^{(N+1) \alpha} y(a+h \tau)\right\}$. Then, by applying the following change of variables: $s \rightarrow \tau s$, we get:

$$
\begin{aligned}
I_{1}(\tau)= & \frac{1}{\Gamma((N+1) \alpha)} \int_{0}^{\tau}(\tau-s)^{(N+1) \alpha-1} \\
& \times{ }_{C} \mathcal{D}_{0, h s}^{(N+1) \alpha} y(a+h s) d s \\
= & \frac{\tau^{(N+1) \alpha}}{\Gamma((N+1) \alpha)} \int_{0}^{1}(1-s)^{(N+1) \alpha-1} \\
& \times{ }_{C} \mathcal{D}_{0, h \tau s}^{(N+1) \alpha} y(a+h \tau s) d s .
\end{aligned}
$$

On the other hand, by taking the $m \alpha$ order derivative to (37) and applying the additive index law given in (9) and the scale change property, we obtain: $\forall \xi \in] 0,1]$,

$$
\mathrm{D}_{0, h \xi}^{m \alpha} y_{N}(a+h \xi)-\mathrm{D}_{0, h \xi}^{m \alpha} y(a+h \xi)=h^{(N+1-m) \alpha} I_{2}(\xi),
$$

where $I_{2}(\xi)=-\mathrm{D}_{0, \xi}^{-(N+1-m) \alpha}\left\{{ }_{C} \mathcal{D}_{0, h \xi}^{(N+1) \alpha} y(a+h \xi)\right\}$. Then, by applying the following change of variables: $s \rightarrow \xi s$, we get:

$$
\begin{aligned}
I_{2}(\xi)= & \frac{\xi^{(N+1-m) \alpha}}{\Gamma((N+1-m) \alpha)} \int_{0}^{1}(1-s)^{(N+1-m) \alpha-1} \\
& \times{ }_{C} \mathcal{D}_{0, h \xi s}^{(N+1) \alpha} y(a+h \xi s) d s .
\end{aligned}
$$


Consequently, it can be deduce from (40) and (43) that: $\forall \xi \in] 0,1]$,

$$
e_{N}^{\infty}(\xi)=h^{(N+1-m) \alpha}\left(\int_{0}^{1} Q_{N}(\tau, \xi) I_{1}(\tau) d \tau+I_{2}(\xi)\right) .
$$

Finally, if we take the absolute value of $e_{N}^{\infty}(\cdot)$ and the following inequalities:

$$
\begin{aligned}
& \left|I_{1}(\tau)\right| \leq M_{N+1} \frac{\tau^{(N+1) \alpha}}{\Gamma((N+1) \alpha+1)} \\
& \left|I_{2}(\xi)\right| \leq M_{N+1} \frac{\xi^{(N+1-m) \alpha}}{\Gamma((N+1-m) \alpha+1)}
\end{aligned}
$$

this proof can be completed.

Finally, by applying Proposition 2 and Theorem 2, the total estimation error for the generalized fractional order Jacobi differentiator is studied.

Corollary 1 Under the hypotheses given in Proposition 2 and Theorem 2, the total estimation error $e_{C}(\xi)=$ $D_{N}^{m \alpha} y^{\varpi}(a+h \xi)-\mathrm{D}_{0, h \xi}^{m \alpha} y(a+h \xi)$ in $D_{N}^{m \alpha} y^{\varpi}(a+h \xi) c a n$ be bounded as follows:

$$
\begin{aligned}
\left|e_{C}(\xi)\right| \leq & h^{(N+1-m) \alpha} M_{N+1} C_{\mu, \kappa, m, \alpha, N}(\xi) \\
& +\frac{1}{h^{m \alpha}} \delta E_{\mu, \kappa, m, \alpha, N}(\xi)
\end{aligned}
$$

where $M_{N+1} C_{\mu, \kappa, m, \alpha, N}(\xi)$ and $\delta E_{\mu, \kappa, m, \alpha, N}(\xi)$ are given in Theorem 2 and Proposition 2, respectively.

In the section of numerical test, the error bounds obtained in Proposition 2 and Theorem 2 will be used to study the influence of the design parameters on the estimation errors $e^{\varpi}(\xi)$ and $e_{N}^{\infty}(\xi)$, respectively.

\subsection{Digital generalized fractional order Jacobi differen- tiator}

From now on, we assume that the noisy signal $y^{\varpi}$ defined in (22) is given in the following discrete case with an equidistant sampling period $T_{s}$ :

$$
y^{\varpi}\left(t_{i}\right)=y\left(t_{i}\right)+\varpi\left(t_{i}\right),
$$

where $T_{s}=\frac{h}{M}, M \in \mathbb{N}^{*}$, and $t_{i}=a+h \xi_{i}$ with $\xi_{i}=\frac{i}{M}$, for $i=0, \cdots, M$.

Since $y^{\varpi}$ is a discrete observation, a numerical integration method should be used to approximate the integral in the generalized fractional order Jacobi differentiator given in (28). In particular, the discrete values of $\phi_{\alpha}^{(\mu, \kappa)}\left(\tau_{j}\right)=\alpha\left(1-\tau_{j}^{\alpha}\right)^{\mu} \tau_{j}^{s}$ should be calculated in (27) for $j=0, \cdots, M$, where $\left.\tau_{j}=\frac{j}{M}, \mu, s \in\right]-1, \infty[$ with $s=\alpha(\kappa+1)-1$. However, if $\mu$ or $s$ is negative, there exists a singular value $\tau_{j}=0$ or at $\tau_{j}=1$. In the following proposition, the generalized fractional order Jacobi differentiator is studied in the discrete case, where four algorithms are provided to overcome the problem of singularity.

Proposition 3 Let $y^{\varpi}$ be the discrete noisy observation defined in (49) and $\tau_{j}=\frac{j}{M}$ for $j=0, \cdots, M$. Then, the digital generalized fractional order Jacobi differentiator denoted by $D_{T_{s}, N}^{m \alpha} y^{\varpi}\left(a+h \xi_{i}\right)$ is defined by considering the following four cases with $s=\alpha(\kappa+1)-1$ : for $i=$ $1, \ldots, M$

Case 1: If $0 \leq \mu, 0 \leq s$, we have:

$$
\begin{aligned}
D_{T_{s}, N}^{m \alpha} y^{\varpi}\left(a+h \xi_{i}\right) \\
:=\frac{1}{2 h^{m \alpha} M}\left\{\sum _ { j = 1 } ^ { M } \left(Q_{N}\left(\tau_{j}, \xi_{i}\right) y^{\varpi}\left(a+h \tau_{j}\right)\right.\right. \\
\left.\left.\quad+Q_{N}\left(\tau_{j-1}, \xi_{i}\right) y^{\varpi}\left(a+h \tau_{j-1}\right)\right)\right\},
\end{aligned}
$$

Case 2: If $0 \leq \mu,-1<s<0$, we have:

$$
\begin{aligned}
D_{T_{s}, N}^{m \alpha} y^{\varpi}\left(a+h \xi_{i}\right) \\
:=\frac{1}{2 h^{m \alpha}}\left\{\sum _ { j = 1 } ^ { M } h _ { j } \left({ }^{s} Q_{N}\left(\hat{\tau}_{j-1}, \xi_{i}\right) y^{\varpi}\left(a+h \tau_{j-1}\right)\right.\right. \\
\left.\quad+{ }^{s} Q_{N}\left(\hat{\tau}_{j}, \xi_{i}\right) y^{\varpi}\left(a+h \tau_{j}\right)\right\},
\end{aligned}
$$

where $\hat{\tau}_{j}=\tau_{j}^{1+s}$ for $j=0, \ldots, M, h_{j}:=\hat{\tau}_{j}-\hat{\tau}_{j-1}$ for $j=1, \ldots, M$, and

${ }^{s} Q_{N}(\tau, \xi)=\frac{\alpha}{1+s}\left(1-\tau^{\frac{\alpha}{1+s}}\right)^{\mu} \sum_{i=0}^{N} E_{i}\left(\tau^{\frac{1}{1+s}}\right) D_{0, \xi}^{m \alpha}\left\{E_{i}(\xi)\right\}$

Case 3: If $0 \leq s,-1<\mu<0$, we have:

$$
\begin{aligned}
& D_{T_{s}, N}^{m \alpha} y^{\varpi}\left(a+h \xi_{i}\right) \\
& :=\frac{1}{2 h^{m \alpha}}\left\{\sum _ { j = 1 } ^ { M } h _ { j } ^ { \prime } \left({ }^{s^{\prime}} Q_{N}\left(\hat{\tau}_{j-1}^{\prime}, \xi_{i}\right) y^{\varpi}\left(a+h \tau_{j-1}\right)\right.\right. \\
& \left.\left.\quad+{ }^{s^{\prime}} Q_{N}\left(\hat{\tau}_{j}^{\prime}, \xi_{i}\right) y^{\varpi}\left(a+h \tau_{j}\right)\right)\right\},
\end{aligned}
$$

where $s^{\prime}=\alpha(\mu+1)-1, \hat{\tau}_{j}=\left(1-\tau_{j}^{\alpha}\right)^{\frac{1+s^{\prime}}{\alpha}}$ for $j=$ 


$$
\begin{aligned}
& 0, \ldots, M, h_{j}^{\prime}:=\hat{\tau}_{j-1}^{\prime}-\hat{\tau}_{j}^{\prime} \text { for } j=1, \ldots, M, \text { and } \\
& { }^{s^{\prime}} Q_{N}(\tau, \xi) \\
& =\frac{\alpha}{1+s^{\prime}}\left(1-\tau^{\frac{\alpha}{1+s^{\prime}}}\right)^{\frac{s+1}{\alpha}-1}\left\{\sum_{i=0}^{N} E_{i}\left(\left(1-\tau^{\frac{\alpha}{1+s^{\prime}}}\right)^{\frac{1}{\alpha}}\right)\right. \\
& \left.\quad \times \mathrm{D}_{0, \xi}^{m \alpha}\left\{E_{i}(\xi)\right\}\right\} .
\end{aligned}
$$

Case 4: If $-1<s<0,-1<\mu<0$, we have:

$$
\begin{aligned}
D_{T_{s}, N}^{m \alpha} y^{\varpi}\left(a+h \xi_{i}\right) \\
:=\frac{1}{2 h^{m \alpha}}\left\{\sum _ { j = 1 } ^ { \lceil \frac { M } { 2 } \rceil } h _ { j } \left({ }^{s} Q_{N}\left(\hat{\tau}_{j-1}, \xi_{i}\right) y^{\varpi}\left(a+h \tau_{j-1}\right)\right.\right. \\
\left.\left.\quad+{ }^{s} Q_{N}\left(\hat{\tau}_{j}, \xi_{i}\right) y^{\varpi}\left(a+h \tau_{j}\right)\right)\right\}+\frac{1}{2 h^{m \alpha}} \\
\quad \times\left\{\sum _ { j = \lceil \frac { M } { 2 } \rceil } ^ { M } h _ { j } ^ { \prime } \left(s^{\prime} Q_{N}\left(\hat{\tau}_{j-1}^{\prime}, \xi_{i}\right) y^{\varpi}\left(a+h \tau_{j-1}\right)\right.\right. \\
\left.\left.\quad+{ }^{\prime} Q_{N}\left(\hat{\tau}_{j}^{\prime}, \xi_{i}\right) y^{\varpi}\left(a+h \tau_{j}\right)\right)\right\} .
\end{aligned}
$$

Proof. The following four cases are considered in the proof.

Case 1: $0 \leq \mu, 0 \leq s$. In this case, the integral in (28) is proper, which can be calculated in the discrete case by the trapezoidal numerical integration method. Hence, (50) is obtained.

Case 2: $0 \leq \mu,-1<s<0$. In this case, the integral given in (28) is improper, which has a singularity at $\tau=0$. In order to overcome this problem, let us apply the following change of variables: $\tau \rightarrow \tau^{\frac{1}{1+s}}$ in (28). Hence, we obtain:

$D_{N}^{m \alpha} y^{\varpi}(a+h \xi)=\frac{1}{h^{m \alpha}} \int_{0}^{1}{ }^{s} Q_{N}(\tau, \xi) y^{\varpi}\left(a+h \tau^{\frac{1}{1+s}}\right) d \tau$,

with

$$
\begin{aligned}
{ }^{s} Q_{N}(\tau, \xi)= & \frac{\alpha}{1+s}\left(1-\tau^{\frac{\alpha}{1+s}}\right)^{\mu}\left\{\sum_{i=0}^{N} E_{i}\left(\tau^{\frac{1}{1+s}}\right)\right. \\
& \left.\times \mathrm{D}_{0, \xi}^{m \alpha}\left\{E_{i}(\xi)\right\}\right\} .
\end{aligned}
$$

Since $y^{\varpi}$ is defined with an equidistant sampling period, let $\hat{\tau}_{j}=\left(\frac{j}{M}\right)^{1+s}$ be the new abscissas such that
$\hat{\tau}_{j}^{\frac{1}{1+s}}=\tau_{j}=\frac{j}{M}$. Hence, the numerical integration steps are $h_{j}=\frac{1}{M^{1+s}}\left(j^{1+s}-(j-1)^{1+s}\right)$, for $j=1, \ldots, M$. Thus, (51) can be obtained by applying trapezoidal numerical integration method to (56).

Case 3: $0 \leq s,-1<\mu<0$. In this case, the integral given in (28) has a singularity at $\tau=1$. In order to overcome this problem, let us first apply the following change of variables: $\tau^{\alpha} \rightarrow 1-\tau^{\alpha}$ i.e. $\tau \rightarrow\left(1-\tau^{\alpha}\right)^{\frac{1}{\alpha}}$ in (28). Hence, we get:

$$
\begin{aligned}
& D_{N}^{m \alpha} y^{\varpi}(a+h \xi) \\
& =\frac{1}{h^{m \alpha}} \int_{0}^{1}{ }^{\mu} Q_{N}(\tau, \xi) y^{\varpi}\left(a+h\left(1-\tau^{\alpha}\right)^{\frac{1}{\alpha}}\right) d \tau,
\end{aligned}
$$

with

$$
\begin{aligned}
{ }^{\mu} Q_{N}= & \alpha \tau^{(\mu+1) \alpha-1}\left(1-\tau^{\alpha}\right)^{\frac{s+1}{\alpha}-1}\left\{\sum_{i=0}^{N} E_{i}\left(\left(1-\tau^{\alpha}\right)^{\frac{1}{\alpha}}\right)\right. \\
& \left.\times \mathrm{D}_{0, \xi}^{m \alpha}\left\{E_{i}(\xi)\right\}\right\} .
\end{aligned}
$$

Since $-1<(\mu+1) \alpha-1<0$ and $\frac{s+1}{\alpha}-1 \geq 0$, the integral obtained in (58) has a singularity at $\tau=0$. Hence, similar to Case 2, let us apply the following change of variables: $\tau \rightarrow \tau^{\frac{1}{1+s^{\prime}}}$ in (58) with $s^{\prime}=(\mu+1) \alpha-1$. Hence, we obtain:

$$
\begin{aligned}
& D_{N}^{m \alpha} y^{\varpi}(a+h \xi) \\
& =\frac{1}{h^{m \alpha}} \int_{0}^{1} s^{\prime} Q_{N}(\tau, \xi) y^{\varpi}\left(a+h\left(1-\tau^{\frac{\alpha}{1+s^{\prime}}}\right)^{\frac{1}{\alpha}}\right) d \tau,
\end{aligned}
$$

with

$$
\begin{aligned}
& { }^{s^{\prime}} Q_{N}(\tau, \xi)=\frac{\alpha}{1+s^{\prime}}\left(1-\tau^{\frac{\alpha}{1+s^{\prime}}}\right)^{\frac{s+1}{\alpha}-1} \\
& \quad \times\left\{\sum_{i=0}^{N} E_{i}\left(\left(1-\tau^{\frac{\alpha}{1+s^{\prime}}}\right)^{\frac{1}{\alpha}}\right) \mathrm{D}_{0, \xi}^{m \alpha}\left\{E_{i}(\xi)\right\}\right\} .
\end{aligned}
$$

Let $\hat{\tau}_{j}=\left(1-\left(\frac{j}{M}\right)^{\alpha}\right)^{\frac{1+s^{\prime}}{\alpha}}$ be the new abscissas such that $\left(1-\hat{\tau}_{j}^{\frac{\alpha}{1+s^{\prime}}}\right)^{\frac{1}{\alpha}}=\tau_{j}=\frac{j}{M}$. Hence, the numerical integration steps are $h_{j}^{\prime}=\left(1-\left(\frac{j-1}{M}\right)^{\alpha}\right)^{\frac{1+s^{\prime}}{\alpha}}-\left(1-\left(\frac{j}{M}\right)^{\alpha}\right)^{\frac{1+s^{\prime}}{\alpha}}$, for $j=1, \ldots, M$. Thus, (53) can be obtained by applying trapezoidal numerical integration method to (60).

Case 4: $-1<s<0,-1<\mu<0$. In this case, the integral given in (28) has two singularities at $\tau=0$ and $\tau=1$. In the case, the integral given in (28) is 
decomposed into two parts:

$$
\begin{aligned}
D_{N}^{m \alpha} y^{\varpi}(a+h \xi)= & \frac{1}{h^{m \alpha}} \int_{0}^{1 / 2} Q_{N}(\tau, \xi) y^{\varpi}(a+h \tau) d \tau \\
& +\frac{1}{h^{m \alpha}} \int_{1 / 2}^{1} Q_{N}(\tau, \xi) y^{\varpi}(a+h \tau) d \tau .
\end{aligned}
$$

Thus, the two integrals in the right side of (62) have singularities at $\tau=0$ and $\tau=1$, respectively. Then, using a similar way as done in Case 2 and Case 3, (55) can be obtained.

Consequently, the estimation error for the digital generalized fractional order Jacobi differentiator $D_{T_{s}, N}^{m \alpha} y^{\varpi}\left(a+h \xi_{i}\right)$ is given in this discrete noisy case by: for $i=1, \ldots, M$,

$$
\begin{aligned}
e_{D}\left(\xi_{i}\right):= & D_{T_{s}, N}^{m \alpha} y^{\varpi}\left(a+h \xi_{i}\right)-D_{0, h \xi}^{m \alpha} y\left(a+h \xi_{i}\right) \\
= & \left(D_{T_{s}, N}^{m \alpha} y^{\varpi}\left(a+h \xi_{i}\right)-D_{T_{s}, N}^{m \alpha} y\left(a+h \xi_{i}\right)\right) \\
& +\left(D_{T_{s}, N}^{m \alpha} y\left(a+h \xi_{i}\right)-D_{N}^{m \alpha} y\left(a+h \xi_{i}\right)\right) \\
& +\left(D_{N}^{m \alpha} y\left(a+h \xi_{i}\right)-D_{0, h \xi}^{m \alpha} y\left(a+h \xi_{i}\right)\right),
\end{aligned}
$$

which contains three sources of errors:

- the discrete noise error contribution $e_{T_{s}}^{\varpi}\left(\xi_{i}\right):=$ $D_{T_{s}, N}^{m \alpha} \varpi\left(a+h \xi_{i}\right)$, which is due to the discrete noise,

- the numerical error $e_{T_{s}}^{\text {trap }}\left(\xi_{i}\right):=D_{T_{s}, N}^{m \alpha} y\left(a+h \xi_{i}\right)$ $D_{N}^{m \alpha} y\left(a+h \xi_{i}\right)$, which is due to the trapezoidal numerical integration method,

- the truncated term error $e_{N}^{\infty}\left(\xi_{i}\right)=D_{N}^{m \alpha} y\left(a+h \xi_{i}\right)-$ $\mathrm{D}_{0, h \xi}^{m \alpha} y\left(a+h \xi_{i}\right)$, which is studied in Subsection 3.2.

Firstly, it is well known that the numerical error $e_{T_{s}}^{\text {trap }}\left(\xi_{i}\right)$ converges to 0 when $T_{s} \rightarrow 0$ [30]. Secondly, using a similar way as done in [14], it can be shown that the discrete noise error contribution $e_{T_{s}}^{\varpi}\left(\xi_{i}\right)$ converges to 0 in mean square when $T_{s} \rightarrow 0$, if the noise $\{\varpi(t), t \in I\}$ is assumed to be a continuous stochastic process satisfying the following conditions:

$\left(C_{1}\right)$ : for any $t_{1}, t_{2} \in I, t_{1} \neq t_{2}, \varpi\left(t_{1}\right)$ and $\varpi\left(t_{2}\right)$ are independent;

$\left(C_{2}\right)$ : the mean value function of $\{\varpi(t), t \in I\}$ denoted by $\mathbb{E}[\cdot]$ is equal to 0 ;

$\left(C_{3}\right)$ : the variance function of $\{\varpi(t), t \in I\}$ denoted by $\operatorname{Var}[\cdot]$ is bounded on $I$.

Note that a zero-mean white Gaussian noise satisfies these conditions.

Consequently, both the numerical error and the discrete noise error contribution can be reduced in the proposed digital fractional order differentiator by decreasing the sampling period $T_{s}$. However, if the computations are performed on a finite precision numerical machine, there are also round-off errors [31]. Hence, the infinite reduction of $T_{s}$ would not lead to arbitrary reduction of estimation errors, since the round-off errors would become too large at some points.

\section{Numerical tests}

In this section, some numerical results are presented to demonstrate the accuracy and the robustness of the generalized fractional order Jacobi differentiator proposed in Proposition 3. It is assumed that $y^{\varpi}\left(t_{i}\right)=$ $y\left(t_{i}\right)+\varpi\left(t_{i}\right)$ is observed on $I=[0, h]$, where the noise $\left\{\varpi\left(t_{i}\right)\right\}$ is simulated from a zero-mean white Gaussian iid sequence, and the variance of $\varpi$ is adjusted such that the signal-to-noise ratio $\mathrm{SNR}=10 \log _{10}\left(\frac{\sum\left|y^{\varpi}\left(t_{i}\right)\right|^{2}}{\sum\left|\varpi\left(t_{i}\right)\right|^{2}}\right)$ is equal to $\mathrm{SNR}=\rho \mathrm{dB}[32]$.

In most cases, the fractional derivatives of a function cannot be analytically calculated, the GrünwaldLetnikov (GL) scheme is usually used to approximate the Riemann-Liouville derivatives in noise-free cases, which is defined as follows $[3,6]: \forall \alpha \in \mathbb{R}$,

$$
{ }_{R} \mathrm{D}_{t}^{\alpha} y(t) \approx \frac{1}{T_{s}^{\alpha}} \sum_{j=0}^{\left\lceil\frac{t}{T_{s}}\right\rceil} w_{j}^{(\alpha)} y\left(t-j T_{s}\right)
$$

where the binomial coefficients can be recursively calculated as follows:

$$
\begin{cases}w_{0}^{(\alpha)}=1, & \text { if } j=0 \\ w_{j}^{(\alpha)}=\left(1-\frac{\alpha+1}{j}\right) w_{j-1}^{(\alpha)}, & \text { else. }\end{cases}
$$

However, this scheme is not robust against noises.

Three kinds of functions are considered in the following examples: a power function, a periodic function and two non-periodic functions, whose Riemann-Liouville fractional derivatives will be estimated by the proposed $\mathrm{d}$ ifferentiator. The Caputo fractional derivatives can be similarly estimated, which is out of the scope of this section. The Riemann-Liouville fractional derivatives of the first two functions can be analytically calculated. However, the ones of the last function are unknown. Hence, the GL scheme is applied in noise-free case in order to verify the efficiency of the proposed differentiator.

The Fractional Order Jacobi Differentiator (FOJD) [14] and the Digital Fractional Order Savitzky-Golay Differentiator (DFOSGD) [13] will also be applied to estimate the Riemann-Liouville fractional derivatives in order to compare with the proposed one. These differentiators 
are based on polynomial approximation methods, which depend on the degree $N$ of the polynomials. In the following examples, same values of $N$ are set for these differentiators and the proposed one. Moreover, the design parameters $\mu$ and $\kappa$ are both set to 0 for FOJD as done in [14] and the design parameter $\theta$ is set to 1 for DFOSGD.

Example 1: Power function. Let $y(t)=-\frac{1}{2} t^{3 \alpha}+\frac{1}{5} t^{4 \alpha}$ on $I=[0,3]$ with $\alpha \in] 0,1$, whose fractional derivatives can be analytically given by Lemma 2 . By taking $T_{s}=$ 0.001 and $\rho=20$, the discrete noisy observation $y^{\varpi}$ can be obtained. Then, the $m \alpha$ order fractional derivative of $y$ will be estimated using $y^{\varpi}$, where the values $m=1$ and $\alpha=0.71$ (resp. $m=2$ and $\alpha=0.95$ ) are set for the proposed differentiator in Proposition 3.

The proposed differentiator depends on three design parameters $N, \mu$ and $\kappa$, which should be chosen to reduce estimation errors. Firstly, since $y$ is a power function, according to Lemma 7, the proposed differentiator does not produce any truncated term error if $N \geq 4$. Secondly, four algorithms are provided in Proposition 3, which are robust against numerical errors. Consequently, $N, \mu$ and $\kappa$ need to be chosen to reduce noise error contributions.

In order to study the influence of the design parameters $N, \mu$ and $\kappa$ on noise error contributions, the noise error bound proposed in Proposition 2 is applied.

(1) By taking $m=1$ and $\alpha=0.71$ (resp. $m=$ 2 and $\alpha=0.95), \mu=\kappa=0$, and $N=$ $4, \ldots, 10$, the variation of $E_{\mu, \kappa, m, \alpha, N}\left(\xi_{i}\right)$ for $\xi_{i}=0.001,0.01,0.02, \ldots, 1$, with respect to $N$ is shown in Fig. 1(a) (resp. Fig. 1(b)). It can be seen that the noise error bound is increasing with respect to $N$. Moreover, it is decreasing as $0.5<\xi_{i} \leq 1$ and is increasing as $0<\xi_{i} \leq 0.5$. Similar results can also be obtained by taking other values of $m, \mu$ and $\kappa$. Consequently, $N=4$ is set in this example.

(2) Now, we fix the value of $N=4$ with $m=1$ and $\alpha=0.71$ (resp. $m=2$ and $\alpha=0.95$ ). For each value of $\xi_{i}$, the contour level set of $E_{\mu, \kappa, m, \alpha, N}\left(\xi_{i}\right)$ with respect to $\kappa$ and $\mu$ can be obtained. These contour level sets vary differently for different values of $\xi_{i}$. In Fig. 2(a) (resp. Fig. 2(b)), the variation of $\left\|E_{\mu, \kappa, m, \alpha, N}\left(\xi_{i}\right)\right\|$ with respect to $\mu$ and $\kappa$ can be observed, where $\mu, \kappa=-0.9,-0.8, \ldots, 1$. Accordingly, smaller values of the noise error bound can be obtained by choosing $-0.5 \leq \kappa \leq 0.5$ (resp. $-0.5 \leq \kappa \leq 0$ ) and $-0.9 \leq \mu \leq 0$. In this example, $\mu=-0.4$ and $\kappa=-0.4$ are chosen to reduce noise error contributions.

Finally, the computation results, the comparison with other methods as well as the corresponding estimation errors are depicted in Fig. $3(m=1, \alpha=0.71)$ and in Fig. $4(m=2, \alpha=0.95)$, respectively.
Example 2: Periodic function. Let $y(t)=\sin (\omega t)$ with $\omega=4$. The Riemann-Liouville fractional derivatives of $y$ can be analytically calculated as follows [5]:

${ }_{R} \mathrm{D}_{t}^{\alpha} y(t)=\frac{\omega t^{1-\alpha}}{\Gamma(2-\alpha)}{ }_{1} \mathrm{~F}_{2}\left(1 ; \frac{1}{2}(2-\alpha), \frac{1}{2}(3-\alpha) ;-\frac{1}{4} \omega^{2} t^{2}\right)$,

where ${ }_{1} \mathrm{~F}_{2}$ is the generalized hypergeometric function. Moreover, we take $I=[0,5], T_{s}=0.001$ and $\rho=10$. ${ }_{R} \mathrm{D}_{t}^{0.51} y$ and ${ }_{R} \mathrm{D}_{t}^{1.02} y$ will be estimated in this example. Hence, $\alpha=0.51$ is set.

Firstly, the design parameters $N, \mu$ and $\kappa$ should be set. On the one hand, different from Example 1, the truncated term error will be produced in this example. Since $N$ corresponds to the truncation order, the truncated ter$\mathrm{m}$ error can be reduced by increasing $N$. On the other hand, similar to Example 1, by studying the noise error bound obtained in Proposition 2, it can be deduced that the noise error contribution is increasing with respect to $N$. In this example, $N=20$ is chosen to reduce the truncated term error. Then, $\mu$ and $\kappa$ are chosen to reduce the noise error contribution. Using a similar way as done in Example 1, it can be deduced that negative values of $\mu$ and $\kappa$ can reduce the noise error contribution. Moreover, by studying the error bound obtained in Theorem 2 , the variation of $\left\|C_{\mu, \kappa, m, \alpha, N}\left(\xi_{i}\right)\right\|$ with respect to $\mu$ and $\kappa$ can be observed in Fig. 5(a) with $m=1$ and Fig. 5(b) with $m=2$, where $\mu, \kappa=-0.9,-0.8, \ldots, 1$. Hence, it can be deduced that negative values of $\mu$ and $\kappa$ can also reduce the truncated term error. Consequently, $\mu=-0.1$ and $\kappa=-0.1$ are chosen both for the cases $m=1$ and $m=2$. Finally, the computation results, the comparison with other methods as well as the corresponding estimation errors are depicted in Fig. 6 with $m=1$ and Fig. 7 with $m=2$, respectively.

In the following two examples, the numerical results obtained by GL scheme are considered as the exact fractional derivatives.

Example 3: Non-periodic function. Let $y(t)=$ $0.01(t+1)^{\frac{2}{3}} \cos (6 t), I=[0,4], T_{s}=0.001$ and $\rho=15$. Then, the $\alpha$ and $2 \alpha$ orders fractional derivatives of $y$ will be estimated with $\alpha=0.7$. By means of similar analysis as done in Example 1 and Example 2, $N=20$, $\mu=-0.2, \kappa=-0.2$ are chosen in the cases of $m=1$ and $m=2$, to reduce truncated term errors and noise error contributions. Finally, the computation results, the comparison with other methods as well as the corresponding estimation errors are depicted in Fig. 8 with $m=1$ and Fig. 9 with $m=2$, respectively.

Example 4: Non-periodic function. Let $y(t)=$ $e^{(-t)} \sin (4 t+1), I=[0,6], T_{s}=0.001$ and $\rho=15$. Then, the $\alpha$ and $2 \alpha$ orders fractional derivatives of $y$ will be 
estimated with $\alpha=0.85$. By means of similar analysis as done previously, $N=22, \mu=-0.2, \kappa=-0.1$ are chosen in the cases of $m=1$ and $m=2$, to reduce truncated term errors and noise error contributions. Finally, the computation results, the comparison with other methods as well as the corresponding estimation errors are depicted in Fig. 10 with $m=1$ and Fig. 11 with $m=2$, respectively.

Consequently, it can be seen in the previous four examples that the results obtained by the proposed fractional order differentiator can be better than the ones obtained by the differentiators: DFOSGD and FOJD.

Finally, by taking different values of $m$ and $\alpha$ we can summarize the influence of the parameters $N, \mu$ and $\kappa$ on the two error bounds obtained in Proposition 2 and Theorem 2 in the following table. According to Tab. 1, the noise error bound is increasing with respect to $N$. Moreover, the intervals of $\mu$ and $\kappa$ are given to reduce the error bounds. Remark that the error bound obtained in Theorem 2 cannot be used to study the influence of $N$ since $M_{N+1}$ is unknown. However, as mentioned previously, the truncated term error can be reduced by increasing $N$.

Table 1

Influence of the design parameters on error bounds

\begin{tabular}{|c|c|c|}
\hline \hline & Noise error bound & Truncated term error bound \\
\hline$N \uparrow$ & $\nearrow$ & \\
\hline$\mu$ & $\in[-0.9,0]$ & $\in[-0.9,0]$ \\
\hline$k$ & $\in[-0.5,0.5]$ & $\in[-0.9,0]$ \\
\hline \hline
\end{tabular}

\section{Conclusions}

In this paper, the fractional order differentiation by integration method was extended by introducing a class of fractional order Jacobi orthonormal functions. Then, the generalized fractional order Jacobi differentiator was proposed by taking the fractional derivative of the truncated fractional order Jacobi orthonormal series expansion of the considered noisy signal. This differentiator was exactly given by an integral formula and depends on a set of design parameters, where the integral can be considered as a low-pass filter. Hence, the proposed differentiator is algebraic and robust against corrupting noises. It can be used to estimate both the RiemannLiouville and the Caputo fractional derivatives with an arbitrary order of the studied signal, without knowing the model defining the signal. By applying the generalized Taylor's formula, some error analysis was provided by giving two error bounds, which were used to study the design parameters' influence. Moreover, three algorithms were provided in discrete noisy case, which are robust against the numerical errors produced by negative design parameters' values. Finally, by comparing with two existing fractional order differentiators: DFOSGD [13] and FOJD [14], numerical results were given to illustrate the accuracy and the robustness of the proposed fractional order differentiator.

\section{Acknowledgement}

This work is supported by the Natural Science Foundation of Hebei Province (A2017203100) in China and the LE STUDIUM RESEARCH PROFESSORSHIP award of Centre-Val de Loire region in France.

\section{References}

[1] B. Ross, The development of fractional calculus 1695-1900, Hist. Math., vol. 4, no. 1, pp. 75-89, 1977.

[2] K.B. Oldham and J. Spanier, The Fractional Calculus, Academic Press, New York 1974.

[3] I. Podlubny, Fractional Differential Equations, Academic Press, New York, NY, USA, 1999.

[4] A.A. Kilbas, H.M. Srivastava and J.J. Trujillo, Theory and Applications of Fractional Differential Equations, vol. 204 of North-Holland Mathematics Studies, Elsevier, Amsterdam, The Netherlands, 2006.

[5] K.S. Miller and B. Ross, An Introduction to the Fractional Calculus and Fractional Differential Equations, Wiley, New York, 1993.

[6] A.M. Concepción, Y.Q. Chen, B.M. Vinagre, D. Xue and V. Feliu-Batlle, Fractional-order Systems and Controls: Fundamentals and Applications, Springer, 2010.

[7] L. Chang, X. Feng, X. Li and R. Zhang, A fusion estimation method based on fractional Fourier transform, Digital Signal Processing, vol. 59, pp. 66-75, 2016.

[8] M.G. El-Mashed, O. Zahran, M.I. Dessouky, M. El-Kordy and F.A. El-Samie, Synthetic aperture radar imaging with fractional Fourier transform and channel equalization, Digital Signal Processing, vol. 23, no. 1, pp. 151-175, 2013.

[9] A. Coronel-Escamilla, J.F. Gómez-Aguilar, L. Torres, M. Valtierra-Rodríguez and R.F. Escobar-Jiménez, Design of a state observer to approximate signals by using the concept of fractional variable-order derivative, Digital Signal Processing, vol. 69, pp. 127-139, 2017.

[10] J.A.T. Machado, Calculation of fractional derivatives of noisy data with genetic algorithms, Nonlinear Dynamics, vol. 57, pp. 253-260, 2009.

[11] J.A.T. Machado, Exploiting sensor redundancy for the calculation of fractional derivatives in the presence of noise, Signal Processing, vol. 92, pp. 204-209, 2012.

[12] D.Y. Liu, G. Zheng, D. Boutat and H.R. Liu, Non-asymptotic fractional order differentiator for a class of fractional order linear systems, Automatica, vol. 78, pp. 61-71, 2017.

[13] D.L. Chen, Y.Q. Chen and D.Y. Xue, Digital Fractional Order Savitzky-Golay Differentiator, IEEE Transactions on Circuits and Systems II: Express Briefs, vol. 58, no. 11, pp. 758-762, 2011.

[14] D.Y. Liu, O. Gibaru, W. Perruquetti and T.M. LalegKirati, Fractional order differentiation by integration and error analysis in noisy environment, IEEE Transactions on Automatic Control, vol. 60, no. 11, pp. 2945-2960, 2015. 
[15] D.Y. Liu and T.M. Laleg-Kirati, Robust fractional order differentiators using generalized modulating functions method, Signal Processing, vol. 107, pp. 395-406, 2015.

[16] D.Y. Liu, Y. Tian, D. Boutat and T.M. Laleg-Kirati, An algebraic fractional order differentiator for a class of signals satisfying a linear differential equation, Signal Processing, vol. 116, pp. 78-90, 2015.

[17] X. Wei, D.Y. Liu and D. Boutat, Non-Asymptotic PseudoState Estimation for a Class of Fractional Order Linear Systems, IEEE Transactions on Automatic Control, vol. 62, no. 3, pp. 1150-1164, 2017.

[18] W. D. Chang, Two-dimensional fractional-order digital differentiator design by using differential evolution algorithm. Digital Signal Processing, vol. 19, no. 4, pp. 660-667, 2009.

[19] E. Diekema, T.H. Koornwinder, Differentiation by integration using orthogonal polynomials, a survey, $J$. Approx. Theory, vol. 164, pp. 637-667, 2012.

[20] M. Mboup, C. Join and M. Fliess, Numerical differentiation with annihilators in noisy environment, Numerical Algorithms, vol. 50, no. 4, pp. 439-467, 2009.

[21] D.Y. Liu, O. Gibaru and W. Perruquetti, Differentiation by integration with Jacobi polynomials, J. Comput. Appl. Math., vol. 235, no. 9, pp. 3015-3032, 2011.

[22] D.Y. Liu, O. Gibaru and W. Perruquetti, Error analysis of Jacobi derivative estimators for noisy signals, Numerical Algorithms, vol. 58, no. 1, pp. 53-83, 2011.

[23] D.Y. Liu, O. Gibaru and W. Perruquetti, Convergence Rate of the Causal Jacobi Derivative Estimator, Curves and Surfaces 2011, LNCS 6920 proceedings, pp. 45-55, 2011.

[24] M. Fliess, Analyse non standard du bruit, C.R. Acad. Sci. Paris, Ser. I, vol. 342, pp. 797-802, 2006.

[25] S. Esmaeili, M. Shamsi and Y. Luchko, Numerical solution of fractional differential equations with a collocation method based on Müntz polynomials, Computers $\&$ Mathematics with Applications, vol. 62, no. 3, pp. 918-929, 2011.

[26] A.H. Bhrawy and M.A. Zaky, Shifted fractional-order Jacobi orthogonal functions: application to a system of fractional differential equations, Applied Mathematical Modelling, vol. 40, no. 2, pp. 832-845, 2016.

[27] M. Abramowitz, and I.A. Stegun, editeurs, Handbook of mathematical functions, GPO, 1965.

[28] Z.M. Odibat and N.T. Shawagfeh, Generalized Taylor's formula, Applied Mathematics and Computation, vol. 186, no. 1, pp. 286-293, 2007.

[29] G. Alexits, Convergence Problems of Orthogonal Series, House of the Hungarian Academy of Sciences, 1961.

[30] A. Ralston, A first course in numerical analysis, McGrawHill, New York, 1965.

[31] R. Butt, Introduction to Numerical Analysis Using MATLAB, Jones \& Bartlett Learning, 2009.

[32] S. Haykin and B. Van Veen, Signals and Systems, 2nd edn. John Wiley \& Sons, 2002.

Xiao-Lin Li earned the Bachelors degree in mathematics and applied mahematics from Anyang Normal University, Anyang China, in 2011. She is currently pursing the Masters degree at the mathematics of computation, Yanshan University, China. Her current research interests include identification and estimation of fractional order systems.
Yi-Ming Chen received his Ph.D. degree in Machinery Design and Theory from Yanshan University, China, in 2002. From 2005 to 2007, he has held a postdoctoral position in Glamorgan University, United Kingdom. Currently, he is a Full Professor in the College of Science of Yanshan University. From 2016 to 2019, he is the LE STUDIUM RESEARCH PROFESSOR in Loire Valley Institute for Advanced Studies, France. His research interests mainly focused on numerical algorithms for differential equations, especially with fractional orders and fractional variational orders, boundary element method in contact problem, robust control and estimation with applications.

Da-Yan Liu earned his Ph.D. degree in Applied Mathematics from University of Lille 1 \& INRIA Lille-Nord Europe in October 2011. Since then, he has held postdoctoral positions in Arts et Métiers ParisTech, France and at King Abdullah University of Science and Technology (KAUST) in Saudi Arabia. From November 2013, he is an Assistant Professor in INSA (French National Institute of Applied Sciences) Centre Val de Loire, where he belongs to Control Team in PRISME Laboratory. His main research interests concern with estimation and identification for integer order linear systems and fractional order linear systems.

Yan-Qiao Wei received the B.S. and M.S. degrees from Taiyuan Normal University and Yanshan University, China, in 2013 and 2016, respectively. He is now working on his Ph.D degree in INSA (French National Institute of Applied Sciences) Central Val de Loire, where he belongs to Control Team in PRESME Laboratory. His interests include identification and estimation of fractional order systems.

Driss Boutat received his Ph.D. degree in Differential Geometry from University of Claude Bernard, Lyon I, France, in 1993. He joined ENSIB in 1997 as Head of the Department of Mathematics, where he earned the Accreditation to Supervise Research (HDR) in 2007. Since 2008, he has been a Full Professor in ENSIB, which developed into the INSA (French National Institute of Applied Sciences) Centre Val de Loire in 2014. Since 2011, Prof. Boutat is the leader of Control Team in PRISME Laboratory. His research interests mainly focused on developing observer normal forms for nonlinear dynamical systems. 


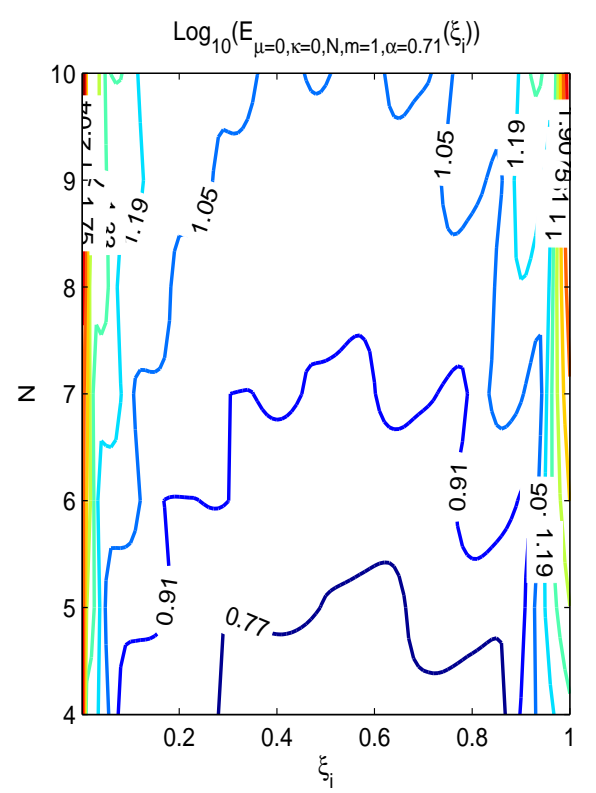

(a) $m=1, \alpha=0.71$.

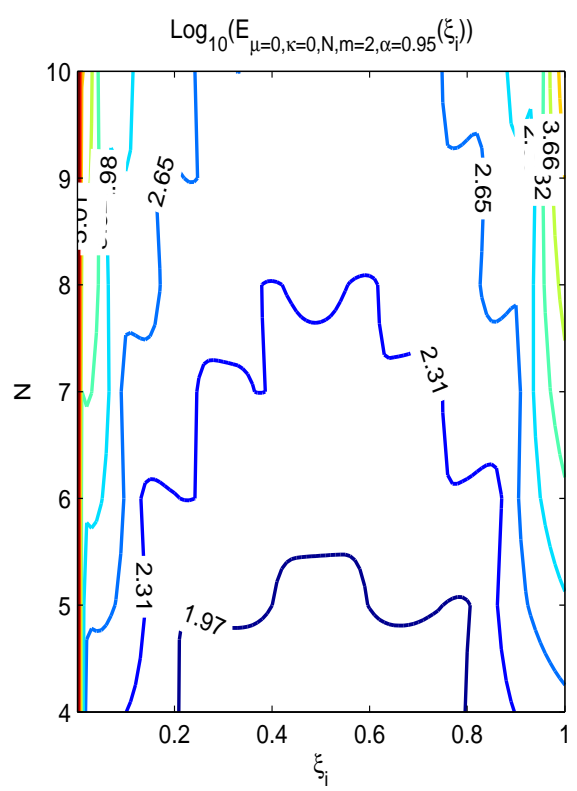

(b) $m=2, \alpha=0.95$.

Fig. 1. Example 1: variation of $\log _{10} E_{\mu=0, \kappa=0, N, m, \alpha}$ with respect to $N$.

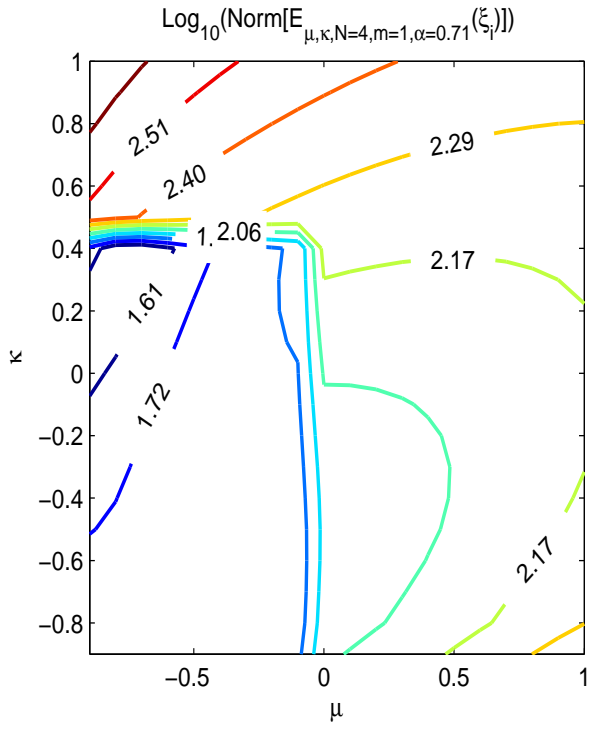

(a) $m=1, \alpha=0.71$.

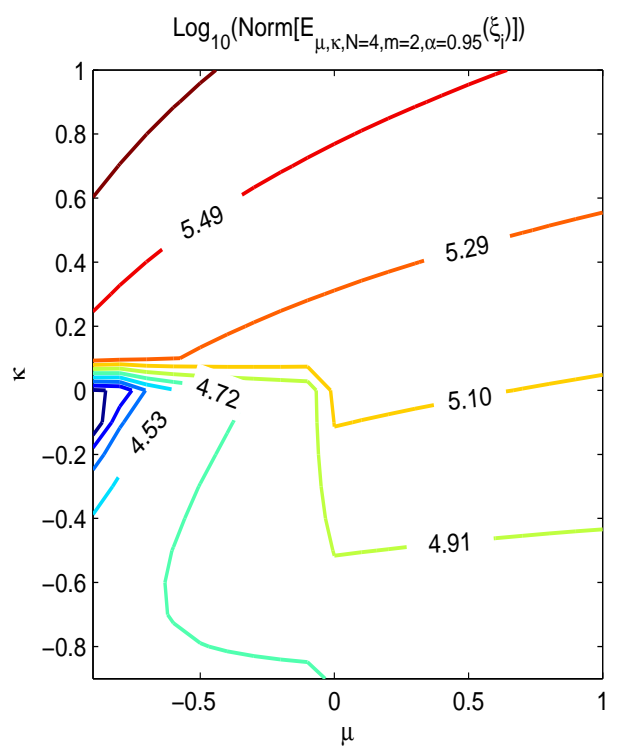

(b) $m=2, \alpha=0.95$.

Fig. 2. Example 1: variation of $\log _{10}\left\|E_{\mu, \kappa, N=4, m, \alpha}\right\|$ with respect to $\mu$ and $\kappa$. 
(b)

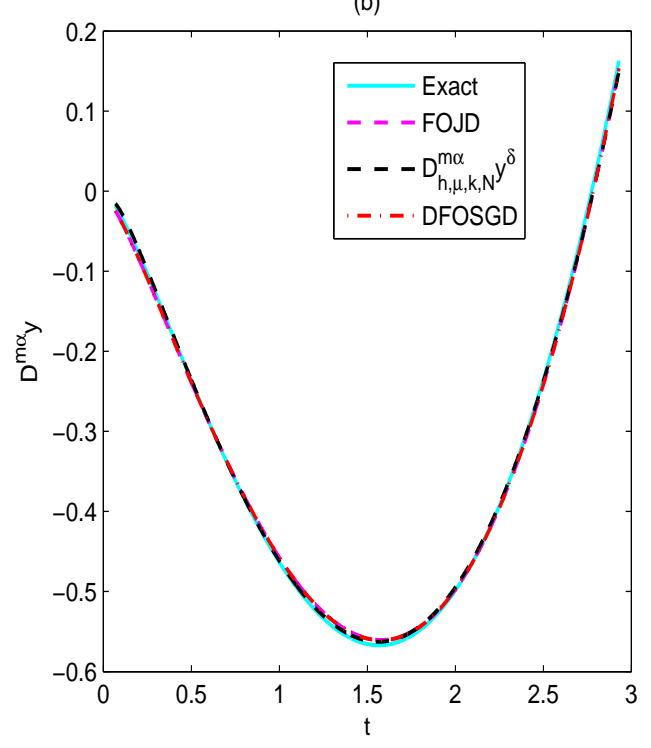

(a) Estimation results.

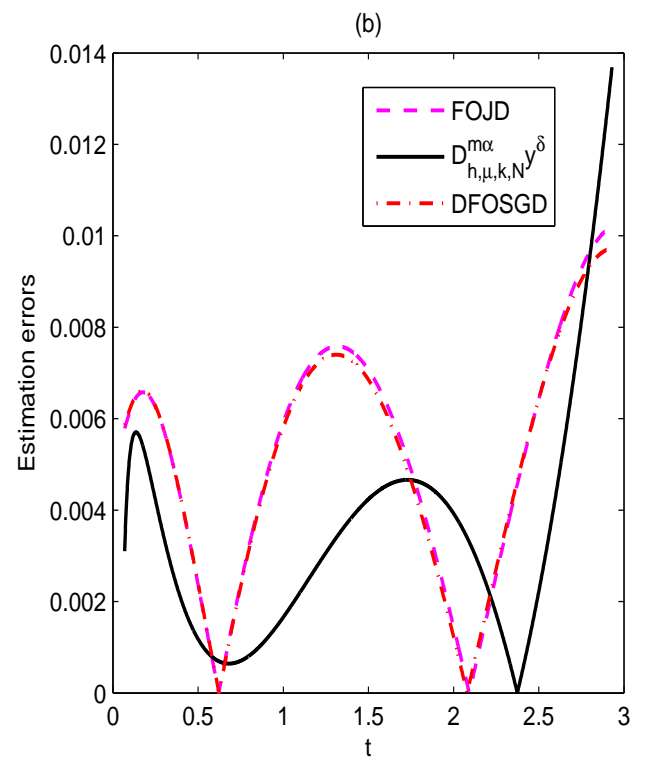

(b) Estimation errors.

Fig. 3. Example 1: estimation results and estimation errors for ${ }_{R} \mathrm{D}_{t}^{0.71}$.

(a)

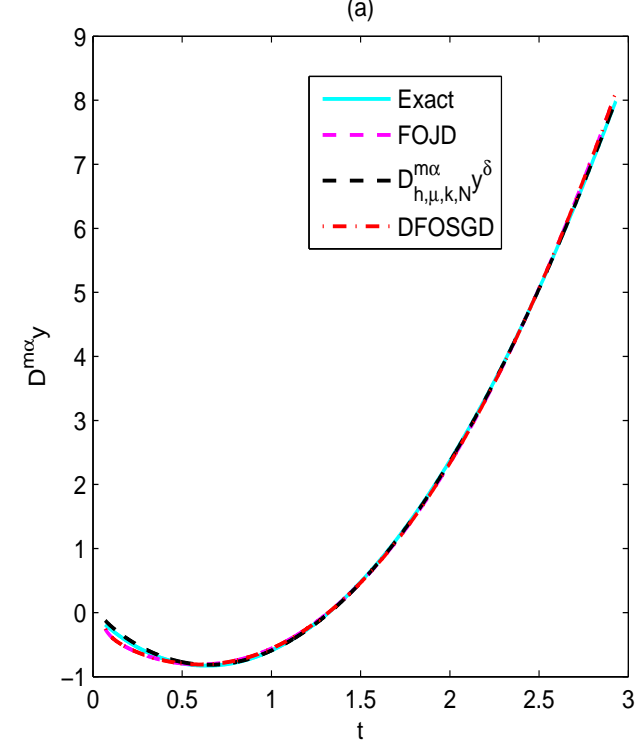

(a) Estimation results.

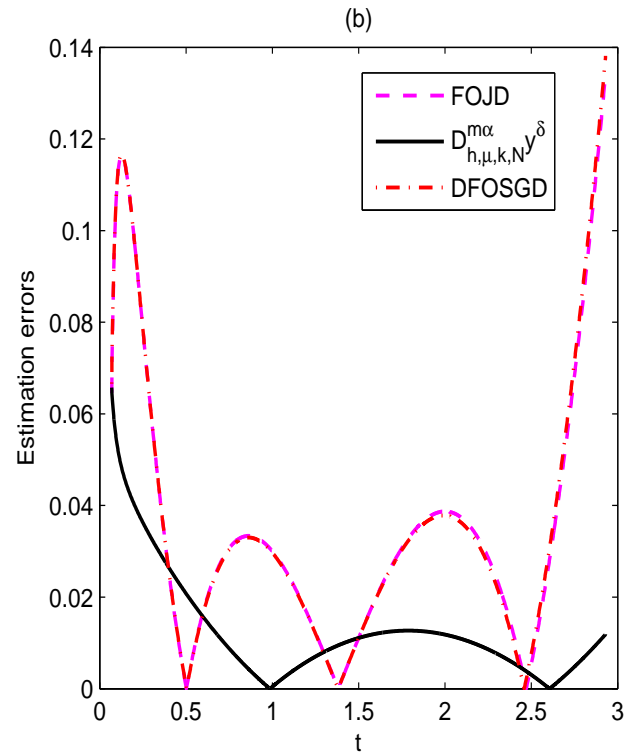

(b) Estimation errors.

Fig. 4. Example 1 : Estimation results and errors of ${ }_{R} \mathrm{D}_{t}^{1.90}$. 


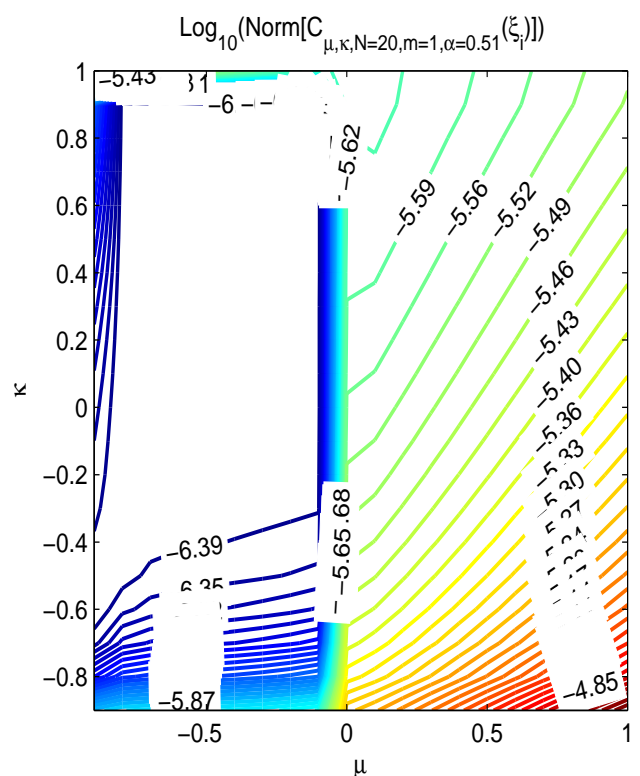

(a) $m=1$.

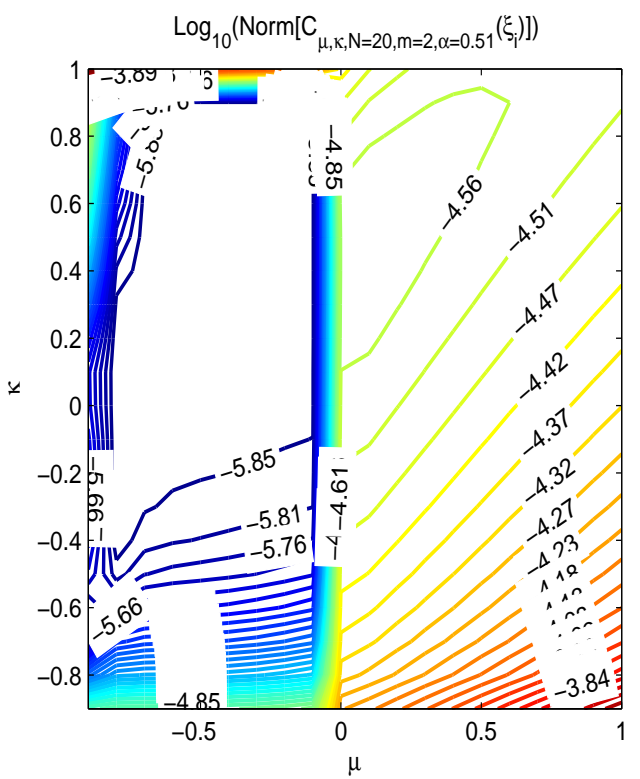

(b) $m=2$.

Fig. 5. Example 2: variation of $\log _{10}\left\|C_{\mu, \kappa, N=20, m, \alpha=0.51}\right\|$ with respect to $\mu$ and $\kappa$.

(a)

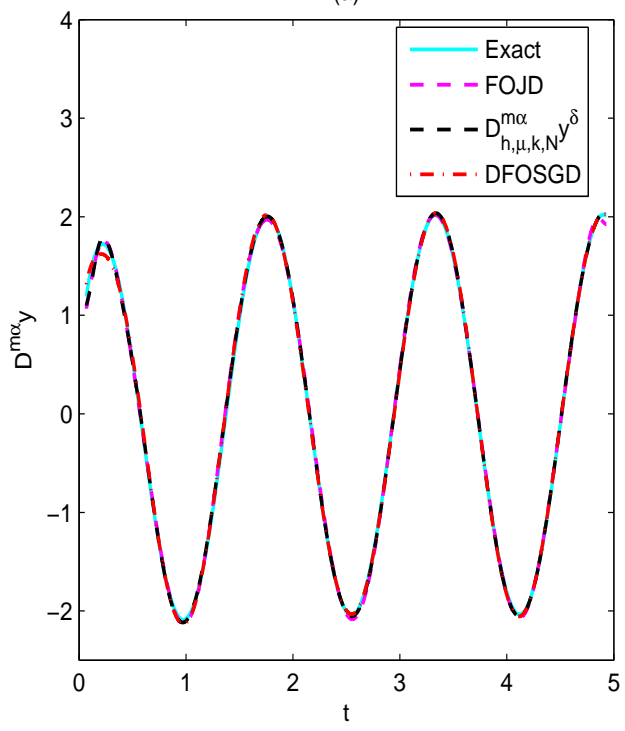

(a) Estimation results.

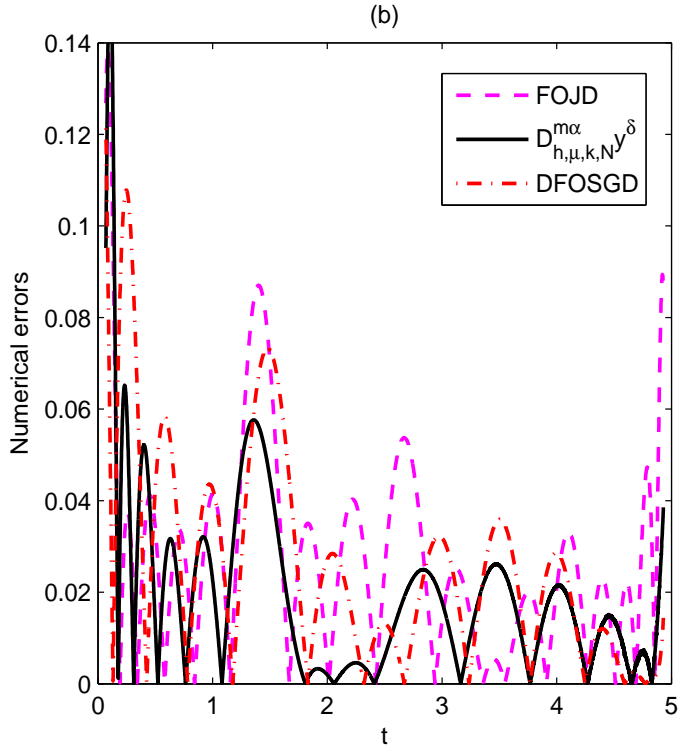

(b) Estimation errors.

Fig. 6. Example 2 : Estimation results and errors of ${ }_{R} \mathrm{D}_{t}^{0.51}$. 
(a)

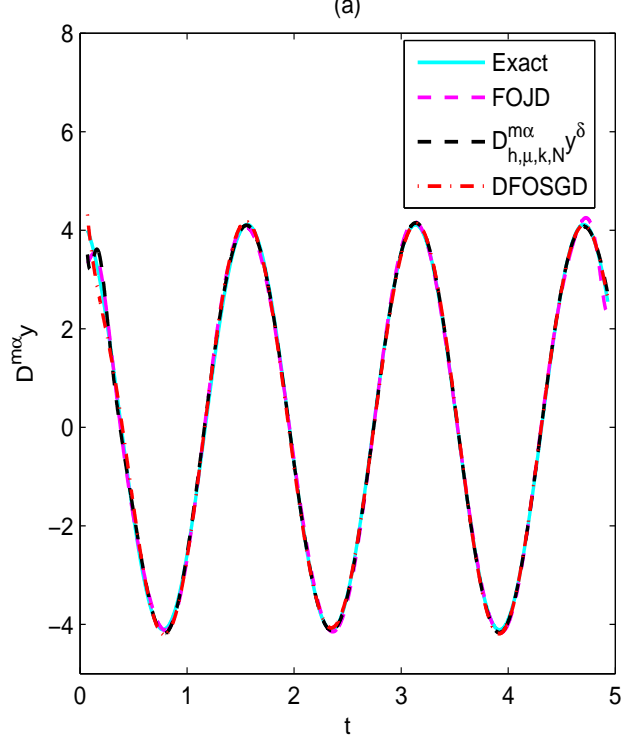

(a) Estimation results. (b)

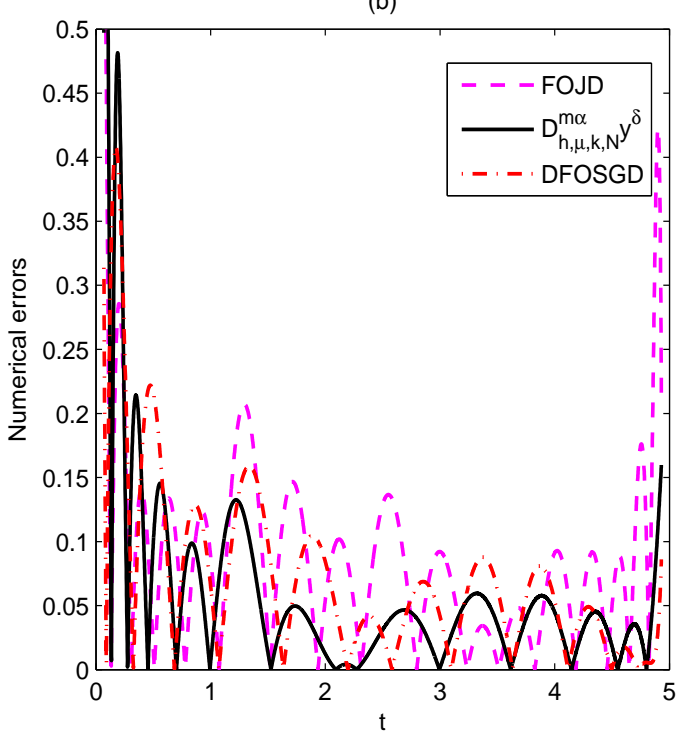

(b) Estimation errors.

Fig. 7. Example 2 : Estimation results and errors of ${ }_{R} \mathrm{D}_{t}^{1.02}$.

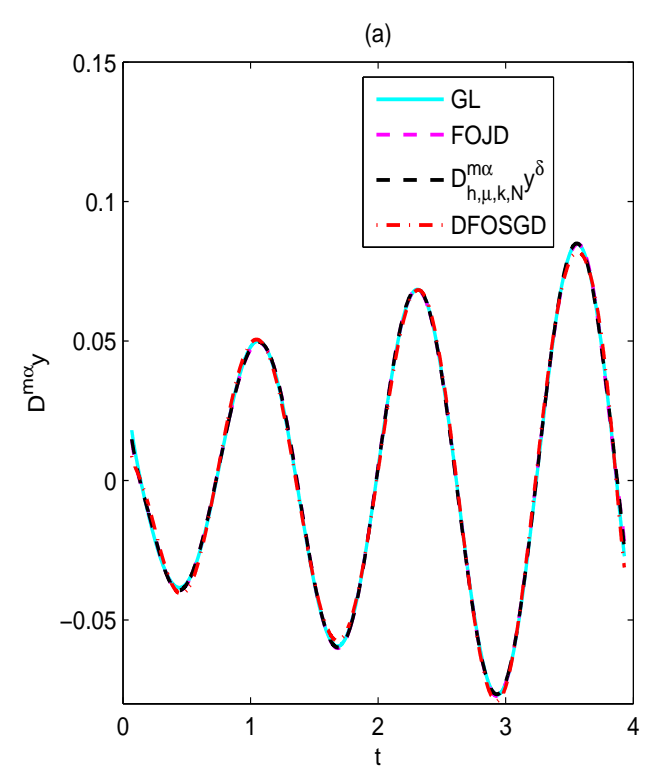

(a) Estimation results.

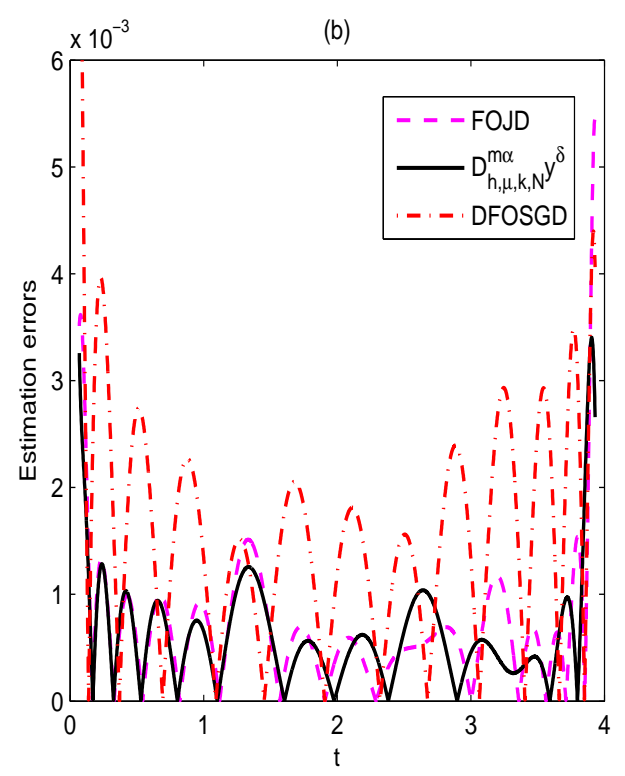

(b) Estimation errors.

Fig. 8. Example 3 : Estimation results and errors of ${ }_{R} \mathrm{D}_{t}^{0.7}$. 
(a)

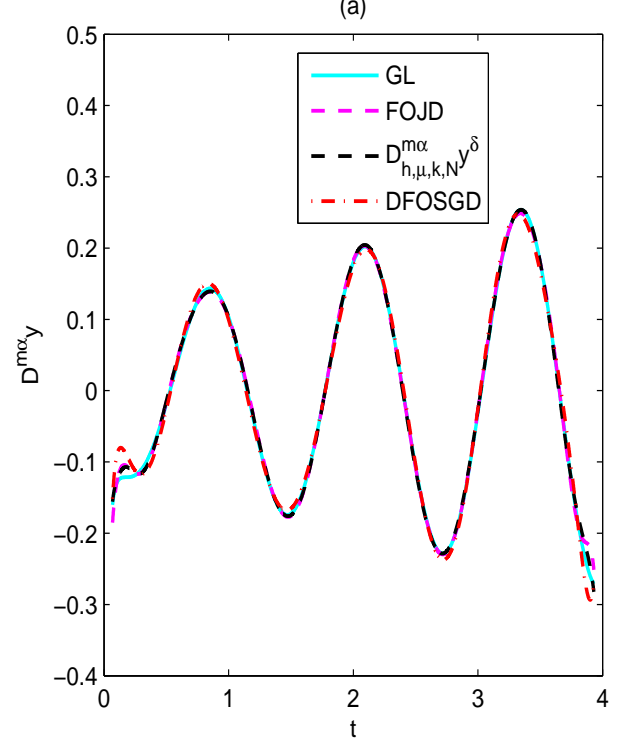

(a) Estimation results.

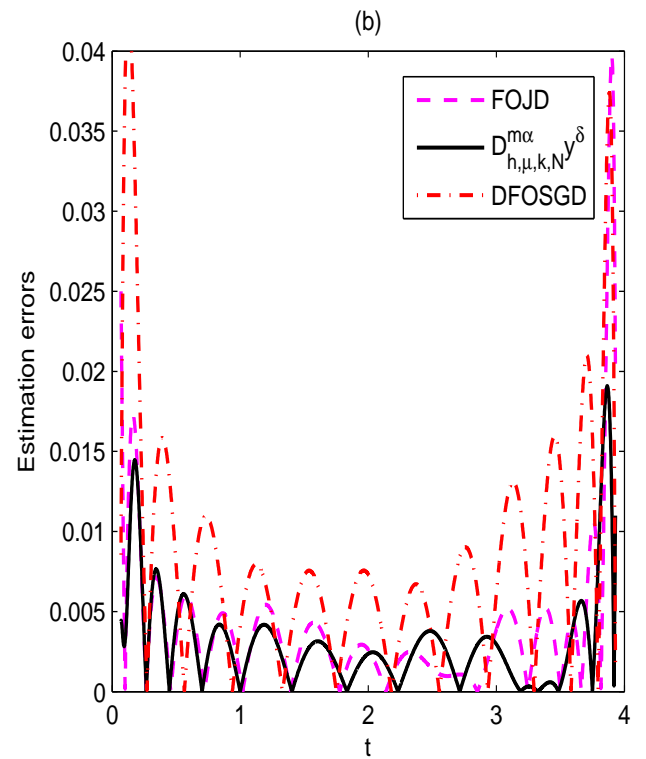

(b) Estimation errors.

Fig. 9. Example 3 : Estimation results and errors of ${ }_{R} \mathrm{D}_{t}^{1.4}$.

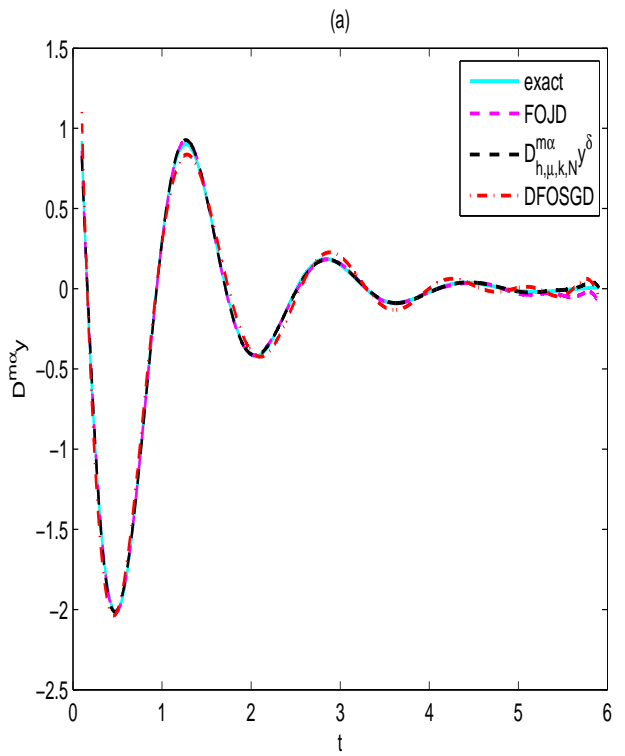

(a) Estimation results.

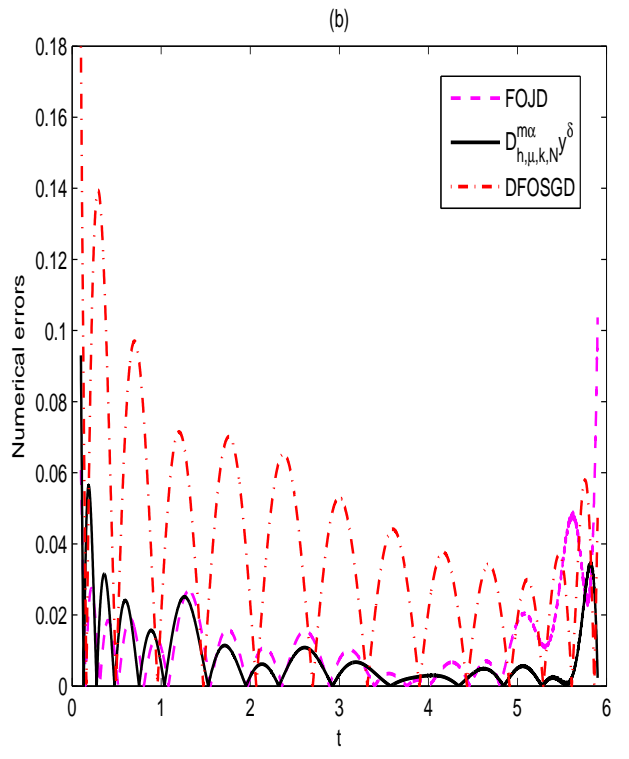

(b) Estimation errors.

Fig. 10. Example 4 : Estimation results and errors of ${ }_{R} \mathrm{D}_{t}^{0.85}$. 


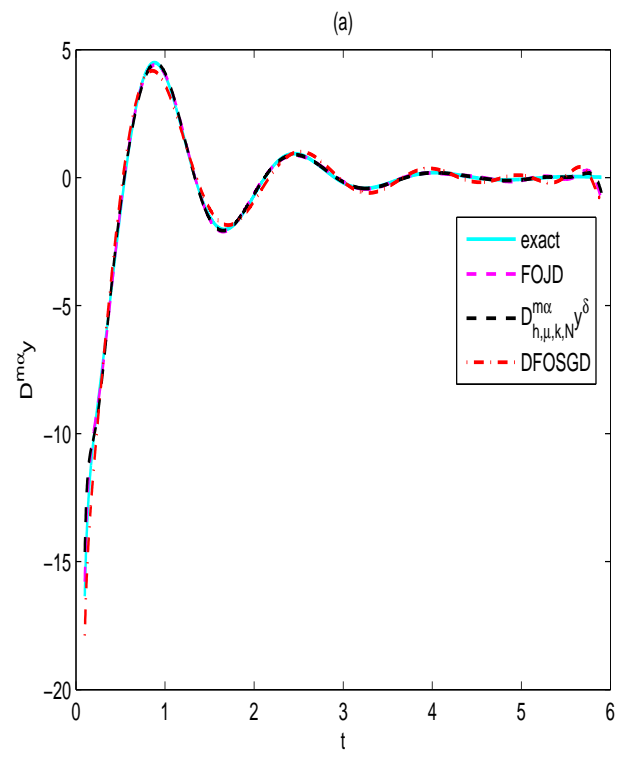

(a) Estimation results.

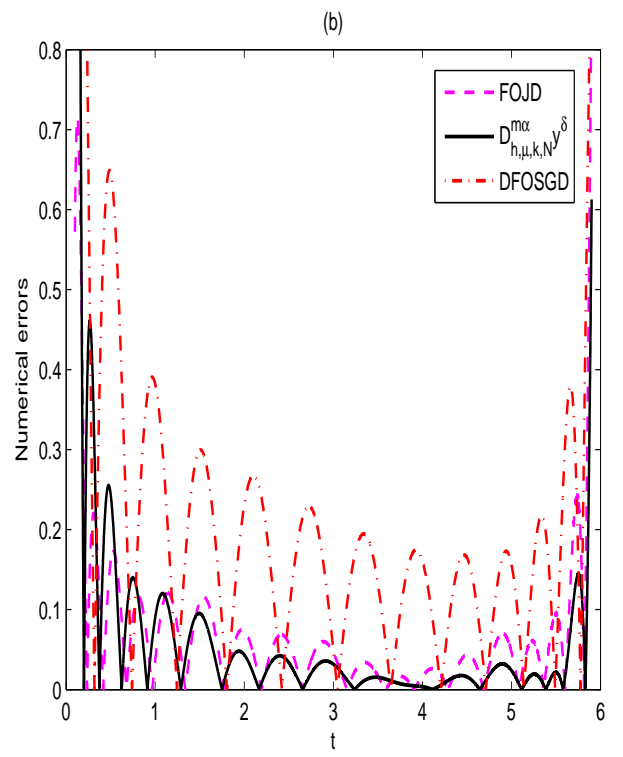

(b) Estimation errors.

Fig. 11. Example 4 : Estimation results and errors of ${ }_{R} \mathrm{D}_{t}^{1.7}$. 